\title{
Impact of Inflation and Unemployment on Economic Growth in Ten (10) Selected Member's States of Economic Community of West Africa States (ECOWAS) (2001-2014)
}

\author{
Gylych Jelilov*, Olanrewaju Joseph Obasa, Abdurahman Isik \\ Economics Department, Nigerian Turkish Nile University, Nigeria
}

Copyright $(2016$ by authors, all rights reserved. Authors agree that this article remains permanently open access under the terms of the Creative Commons Attribution License 4.0 International License

\begin{abstract}
The study set three major objectives which include determine the relationship between economic growth, inflation and unemployment, analyses the effects of inflation in ten (10) selected members of ECOWAS and assess the effects of unemployment in the selected members states. Secondary data obtained from the Member's State National Statistics offices was used for the paper. The study used a model in which inflation and unemployment were the dependent variable and independent variables. The analytical technique used includes ordinary least square (OLS) technique, F-test. The paper showed that monetary and fiscal policy were effective in the control of the inflation and unemployment since the coefficient of determination $\left(\mathrm{R}^{2}\right.$ $=0.50$ or $50 \%$ was significant. This was re-confirmed by the F-test value (4.91). The paper recommends a policy redirection to improve output in the ten (10) selected member's states; this will occur by making efforts to increase productivity, which will lead to reduction in unemployment and inflation. To curb the surging rate of unemployment, efforts must be put in place to achieve a labour intensive method of production instead of concentrating on the capital intensive method which will take away jobs that individuals can do. Furthermore, there must be concrete efforts to ensure that the porous borders in the ten (10) selected members states are well managed to increase volume of economic activities among the members' States, which is very pivotal for the reduction of unemployment and inflation; thereby improving the level of local production.
\end{abstract}

Keywords ECOWAS, Inflation, Unemployment and Economic Growth

\section{Introduction}

The economic and political landscape of any country hinges on inflation and unemployment because of the changes it can ignite in the system. Actually among the two, the tradeoff is curtailed. Therefore, on the long run no tradeoff will occur; thus, they both may now move in the same direction; however this may not occur at the same time (Wallich, 1979).

Overtime, economists have tried to establish the relationship between inflation and unemployment; however these two variables are linked together economically. The relationship that exist between them are inversely correlated; therefore when unemployment is high, inflation is low and otherwise (Umaru \& Zubairu, 2012).

In any economy, inflation and unemployment are always on the "front burner"; all economies will always intend to keep them both on a low rate mostly on a single-digit rate because this will tend to bring about stability in the macroeconomic policies of the country. This stability is pivotal to effectively achieve growth and development in the economy and also the attainment of its set out goals and objectives of its economic policies (Orji, Orji-Anthony, \& Okafor, 2015).

When money supply is altered, this in turn leads to inflation. Therefore, when money supply is increased, it will have a multiplier effect on the price of goods and services in the economy which will lead to its increase also. Hence, inflation is the upward movement in the prices of goods and services. The classical economist defined the long term Phillips curve to be the natural rate of unemployment in an economy. It states that on the long run, inflation and unemployment are not meant to have a relationship (Phillips, 1958)(Friedman, 1968).

Therefore, if employment rate is less than the natural rate, thus inflation rate will exceed the limits of expected rate and therefore the unemployment rate is higher than the acceptable limit, therefore the inflation rate will be less than the expected rate (Phillips, 1958) (Friedman, 1968).

Inflation as explained by the Keynesian implies the supply 
of money that keeps rising. They focus mainly with institutional crises that people face, when the industries raise the prices of goods and services. Industries make significant yields when they increase the prices of their goods and services. Furthermore, the Central Bank increases the supply of money to ensure the continuous functionality of the economy (Phillips, 1958)(Friedman, 1968).

Inflation and unemployment are very critical to the economic growth and development of any economy. These two (2) factors are mainly used to examine the level of poverty in developing economies. Therefore, countries are encouraged to continually increase their level of produce because this will help to cushion the effect of inflation in the economy. Also, increase in the level of goods and services will improve the standard of living and therefore create social harmony within the country.

The inflation rate in the economy of Nigeria and the other selected member states in ECOWAS have in recent years been fluctuating mainly due to the inconsistencies in the Real Gross Domestic Product (RGDP) (CBN, 2004).Also, other economic indicators such as unemployment rate are indicators of an ailing economy; this study is conducted to examine the impact of inflation on unemployment in the ten (10) selected member's states.

The broad objective of the study is to empirically examine the impact of unemployment, inflation on economic growth in the ten (10) selected member's states.

The specifically objectives are:

(i) Determine the relationship between economic growth, inflation and unemployment.

(ii) Analyses the effects of inflation in the ten (10) selected member's states.

(iii) Assess the effects of unemployment in the ten (10) selected member's states.

\section{Literature Review}

\subsection{The Concept of Inflation and Unemployment}

Inflation is commonly seen to be a situation in the economy when the money supply is growing faster than the production of new goods and services in the same economy (Hamilton, 2001). Inflation is further defined to be the general price increase in goods and services over a particular time mainly for a long period (Balami, 2006). However, the definition of inflation, the cause of inflation and its effects on the economy depends on the economic school of taught the economist belongs this is evident in the various definitions of inflation that has emerged over time. Similarly, arguments have occurred among economists in trying to distinct Inflation from an economic occurrence which result in price increase of goods and services at a certain time or when there is an upward movement in prices of economic goods and services in a specific slender group (Piana, 2001). This argument has led to economist having diverging views about inflation, the cause of inflation and unemployment and their resultant effects on the economy and recommendations.

Classical economist which are regarded as mainstream economist believe that unemployment and inflation are a short-run phenomenon which takes care of itself in the long-run because of the assumption of flexible prices and wages. The monetarist sees inflation as limited available resources relative to available money in circulation i.e money supplied is greater than output and suggested control in money supply the monetary authorities as panacea for inflation (Jhingan 2008). The Keynesian suggested government intervention through payment of unemployment benefits in order to keep the consumer spending constant since it is their belief that inflation occurs when the aggregate demand exceeds the aggregate production of output i.e, total expenditure is greater than available output and the presence of inflation causes further inflation which is referred to as cost push inflation. To them, there is always a trade-off between inflation and unemployment. They further stipulate that unemployment has the capacity to reduce aggregate output as it reduces income and limits consumption because consumption decision of consumers is based on current income available to them. Stating further that aggregate demand falls owing to declining private investment that make firms to produce below capacity and as a result, need fewer workers thereby leading to unemployment. The new neoclassicals on the other hand see this unemployment (involuntary unemployment) as an opportunity for firms to make further profit by means of wage cut to a low level without minding if the employed workers would resist such wages reduction since the unemployed stand willing to accept lower wages (Hover 2016). The Rational Expectation philosophy on inflation and unemployment is rooted in the fact that "People are forward-looking and rational as a result, they incorporate this into their expectations, and the future tends to follow their expectation". They believe that firms and workers depends not just on the past information but equally make some predictions about the future therefore, the labour market is believed to be in equilibrium often time and leave unemployment at it natural rate. Finally, the Rational Expectations agreed that indeed, government can intervene in reducing unemployment rate, but such intervention always lead to inflation or what they termed as "higher price" (Study.Com,2016) This conclusion is similar to the Philip Curves which expresses an inverse relationship between Unemployment and inflation.

The empirical macroeconomists find inflation and unemployment as essentially challenging and therefore a lot of studies have been conducted in more advanced countries. Some suggestions arise on if possible to stabilize without recession. Also, some models have suggested that stabilization might be expansionary especially in countries where high inflation is prevalent. However, stabilization without recession is most likely unachievable (Kamin \& Klau, 1998).

Inflation is well known to be a situation in the economy when the money supply is growing faster than the production of new goods and services in the same economy (Hamilton, 
2001). Inflation is further defined to be the general price increase in goods and services over a particular time mainly for a long period (Balami, 2006).

Arguments have occurred among economists in trying to distinct Inflation from an economic occurrence which result in price increase of goods and services at a certain time or when there is an upward movement in prices of economic goods and services in a specific slender group (Piana, 2001).

The International Labour Organmization (ILO) defines the unemployed as those relevant population in the economic activies among the entire populace that are willing to work but have no work. This also comprises of those who left work willingly (World Bank, 1998).

The Phillips curve is divided into some assumptions: the negative, the natural hypotheses and the positive hypothesis. The explanation of the link between inflation and unemployment has gone through some phases since the conclusion of World War II. The first phase was the assent of the Phillips hypothesis (Friedman, 1976).

Phillips discussed that there was a consistent negative link between the level of unemployment and the rate of changes in wages. The reduction in wages is associated with high levels of unemployment while increase in wages is associated with low level of unemployment. The change in wages is associated to changes in prices of goods and services by allowing for a rise in the level of productivity and treating the price excesses over wage cost through a consistent mark-up factor (Phillips, 1958) (Friedman, 1976).

A research was conducted on inflation and unemployment in the EU for 1998-2007; it was established that the simple linear correlation coefficient between inflation and unemployment is negative. Invariably, it leads to the conclusion that their relationship is not excessive and negative (Popovic \& Popovic, 2009).

Another research was conducted using Nigeria's economic situation to examine the trade-off between inflation and unemployment in less developed economies. OLS model was used and it was observed that there was no trade-off between the two factors. This further showed a case of stagflation in the economy of Nigeria (Abachi, 1998).

Also, there was a test on the connection between money, inflation and output through the usage of cointegration and granger-causality test analysis. The research showed that there was non-availability of a cointegrating vector in the series used. Granger concluded that money supply causes output and inflation. This therefore ascertains that monetary policy has a major input on price stability in the economy of Nigeria simply because the variation in price level is majorly influenced by money supply. Therefore, inflation in the economy is mainly a monetary issue (Omoke \& Ugwuanyi, 2010).

Inflation has grievious effect especially on fixed incomes in an economy, this has drastic effect on their standard of living due to reduction in real income, savings and capital formation (Buhari, 1987). Economic growth is drastically affected by inflation and therefore limits economic development in a country, this leads to the creation of unrest among the populaces (Adamson, 2000). This makes it evident that inflation in the ten (10) selected member's states are mainly monetary issues. This can be further seen empiracally in the money-price-output hypothesis in the ten (10) selected member's states.

\subsection{Regional Economic Integration in ECOWAS}

The sub-region of West Africa has improved recently in its growth rate, though it has endured some past decades of low economic activities and impaired civil war. However, things have changed in recent times for the better specifically reaching a growth rate of $7 \%$ in 2012. ECOWAS is very purposeful in its drive for economic integration and this is supported by its vast population and improved private partnership (Sack, 2016).

\section{Methodology}

\subsection{Study Area}

This will cover ten (10) selected member's states in Economic Community of West Africa States (ECOWAS) which was established in 1975; it comprises of countries in the West African sub-region. The selected countries are: Nigeria, Benin Republic, Cape Verde, Cote d'ivoire, Gambia, Ghana, Guinea Bissau, Mali, Niger and Senegal. The choice of ECOWAS is informed by the attempt of ECOWAS authority as a regional block to convert the 15 member States to a common market by introduction of single currency and other sundry socio-economic policies in the 2020. This makes the research necessary to determine ahead of the implementation of this policy to see the behavior of the regional economy as one.

\subsection{Method of Data Collection}

This study made use of secondary data from member's state National Statistics offices, World Bank and International Monetary Fund database.

\subsection{Method of Data Analysis}

The Model analysis was conducted using the Ordinary Least Square (OLS) regression. Data on inflation and unemployment rate was used for the estimation of parameters of the Model. The coefficient of determination $\left(\mathrm{R}^{2}\right)$, T-test, F-test and Durbin Watson (DW) statistics were used. Also, to confirm the level of serial correlation among variables, Durbin Watson statistics was used.

\subsection{Linear Regression Model}

This study adopts the Okun's model with a bit of adjustment and modifications to incorporate economic growth into the model. The economy as the endogenous variable is represented by GDP while inflation and 
unemployment are exogenous variables in the model. The application of the Okun's model which is the reduced form of Philips Model is that, not only the short-run trade-off between inflation and unemployment has become one of the most important tools in the design and implementation of monetary policy by most monetary authorities; it is equally used to asses inflationary pressures and to forecast inflation (Llaudes ,2005)

The model is explained as:

$$
\begin{gathered}
\text { GDP }=\text { fINFLA }) \\
\text { UNEMPL }=\mathrm{f}(\text { INFLA })
\end{gathered}
$$

Hence,

$$
\mathrm{GDP}=\alpha+\beta \text { INFLA }+\mu
$$

Where GDP is the Gross domestic Product (GDP), INFLA is the inflation rate and UNEMPLO is unemployment rate.

$\alpha$ and $\beta$ - Parameters

$\mu$ - Error term (white noise)

\subsection{A' Priori Expectation}

It is anticipated that: $\alpha>0, \beta>0$

\subsection{Model II (Granger causality model)}

$$
\begin{gathered}
\text { GDP }=\Sigma \alpha \text { GDP }_{\mathrm{t}-1}+\Sigma \alpha \text { INFLA }_{\mathrm{t}-1}+\mu_{\mathrm{t}} \\
\mathrm{UNEMPL}=\Sigma \alpha \mathrm{UNEMPL}_{\mathrm{t}-1}+\Sigma \alpha \mathrm{INFLA}_{\mathrm{t}-1}
\end{gathered}
$$

\subsection{Decision Rules}

The decision rule under causality models for equation (3) is to test for null hypothesis to know that the estimated coefficients are equal to zero( 0$)$ at an appropriate level of significance or to make use of the rule of thumb that if $\mathrm{t}$-statistic is at least 2 the null hypothesis is rejected otherwise accepted. Therefore, Equation (3) INFLA causes UNEMPL and impact on economic growth if $\mathrm{H}_{0}$ : $=0$ is rejected.

Therefore, if the estimates of the parameter come up with signs or sizes that do not go in accordance to economic assumptions, hence should be rejected, otherwise if there is a good reason to accept that in the particular instance, the principles of economic assumptions do not stand.

\subsection{Model Specification}

$$
\mathrm{GDP}=\mathrm{F}(\mathrm{X})+\mathrm{U}_{\mathrm{t}}
$$

Where

$$
\begin{array}{ll}
\text { GDP } & =\text { Dependable }(\mathrm{Y}) \\
\mathrm{INFL} & =\text { Inflation rate }(\mathrm{X}) \\
\mathrm{U}_{\mathrm{t}} & =\text { Stochastic (error) variable }
\end{array}
$$

In model above, where the GDP is the dependent variable and Inflation is the independent variable.

\subsection{Nigerian Econometric Model}

Table 1. Regression Data for Nigeria

\begin{tabular}{|c|c|c|c|}
\hline Year & $\begin{array}{c}\text { GDP (US } \\
\text { \$ Million) }\end{array}$ & $\begin{array}{c}\text { Unemployment } \\
\text { rate (\%) }\end{array}$ & $\begin{array}{c}\text { Inflation rate } \\
(\%)\end{array}$ \\
\hline 2001 & $70,976.56$ & 13.6 & 16.5 \\
\hline 2002 & $95,063.46$ & 12.6 & 12.1 \\
\hline 2003 & $108,794.63$ & 14.8 & 23.8 \\
\hline 2004 & $141,260.66$ & 13.4 & 10.0 \\
\hline 2005 & $180,502.01$ & 11.9 & 11.6 \\
\hline 2006 & $233,859.81$ & 12.3 & 8.6 \\
\hline 2007 & $267,663.48$ & 12.7 & 6.6 \\
\hline 2008 & $334,580.51$ & 14.9 & 15.1 \\
\hline 2009 & $272,536.01$ & 19.7 & 12.1 \\
\hline 2010 & $369,062.40$ & 21.4 & 12.1 \\
\hline 2011 & $411,743.80$ & 23.9 & 10.8 \\
\hline 2012 & $460,953.92$ & 27.4 & 12.9 \\
\hline 2013 & $514,964.76$ & 24.7 & 8.8 \\
\hline 2014 & $575,304.15$ & 7.5 & 8.1 \\
\hline
\end{tabular}

Source: National Bureau of Statistics, Nigeria

Table 2. Log of Regression Data for Nigeria

\begin{tabular}{|c|c|c|c|}
\hline Year & GDP $(\%)$ & $\begin{array}{c}\text { Unemployment } \\
\text { rate }(\%)\end{array}$ & $\begin{array}{c}\text { Inflation rate } \\
(\%)\end{array}$ \\
\hline 2001 & 4.85 & 1.13 & 1.22 \\
\hline 2002 & 4.98 & 1.10 & 1.08 \\
\hline 2003 & 5.04 & 1.17 & 1.38 \\
\hline 2004 & 5.15 & 1.13 & 1.00 \\
\hline 2005 & 5.26 & 1.08 & 1.06 \\
\hline 2006 & 5.37 & 1.09 & 0.93 \\
\hline 2007 & 5.43 & 1.10 & 0.82 \\
\hline 2008 & 5.52 & 1.17 & 1.18 \\
\hline 2009 & 5.44 & 1.29 & 1.08 \\
\hline 2010 & 5.57 & 1.33 & 1.08 \\
\hline 2011 & 5.61 & 1.38 & 1.03 \\
\hline 2012 & 5.66 & 1.44 & 1.11 \\
\hline 2013 & 5.71 & 1.39 & 0.95 \\
\hline 2014 & 5.76 & 0.88 & 0.91 \\
\hline
\end{tabular}

Source: Own computation

$$
\mathrm{GDP}=\mathrm{F}\left(\mathrm{X}_{1}+\mathrm{X}_{2}\right)+\mathrm{U}_{\mathrm{t}}
$$

$$
\begin{array}{lll}
\text { GDP } & = & \text { Dependable }(Y) \\
\text { UNEMPL } & = & \text { Independent }\left(\mathrm{X}_{1}\right) \\
\text { INFLA } & = & \text { Independent }\left(\mathrm{X}_{2}\right)
\end{array}
$$

The model above was built to ascertain the impact of unemployment and inflation on economic growth as well as effect of inflation variable on Unemployment.

$$
\begin{aligned}
\text { GDP }_{\mathrm{t}-1}= & 2.93-1.58 \text { INFLA }_{\mathrm{t}}+3.74 \text { INFLA }_{\mathrm{t}-1}+\mathrm{U}_{\mathrm{t}} \\
& (1.4439)(-0.984)(5.206)
\end{aligned}
$$

T-statistics are in parenthesized

$\mathrm{R}^{2}=0.754 \quad$ Adjusted $\mathrm{R}^{2}=0.709$

F-Statistics $=16.87 \quad \mathrm{D}-\mathrm{W}=1.60$ 
The above equation (8) shows that a unit increase on economic growth (GDP) in the previous year would bring about -1.58 decrease in the prices of goods and services in the present year and 3.7 increase in the prices of goods and services in the previous years.

UNEMPLOt=1.055+0.523UNEMPLOt-1-0.421INFLAt-1+Ut m

$$
\text { (1.055) (3.027) }
$$

$\mathrm{T}$-statistics are in parenthesized

$\mathrm{R}^{2}=0.43 \quad$ Adjusted $\mathrm{R}^{2}=0.394$

F-Statistics $=4.91 \mathrm{D}-\mathrm{W}=1.45$

The above equation (9)shows that a unit increase on unemployment rate would bring about -0.421 decrease in inflation rate in the short-run when there is tradeoff but in the long-run when tradeoff disappearance, a unit increase on unemployment rate

\section{3..9.2. Benin Republic econometric Model}

Table 3. Regression Data for Benin Republic

\begin{tabular}{|c|c|c|c|}
\hline Year & $\begin{array}{c}\text { GDP (US } \\
\text { \$ Million) }\end{array}$ & $\begin{array}{c}\text { Unemployment } \\
\text { rate (\%) }\end{array}$ & $\begin{array}{c}\text { Inflation } \\
\text { rate }) \%)\end{array}$ \\
\hline 2001 & $2,499.27$ & 0.7 & 4.0 \\
\hline 2002 & $2,807.65$ & 0.7 & 2.5 \\
\hline 2003 & $3,557.22$ & 0.9 & 1.5 \\
\hline 2004 & $4,050.86$ & 1.3 & 0.9 \\
\hline 2005 & $4,358.01$ & 1.3 & 5.4 \\
\hline 2006 & $4,705.08$ & 1.1 & 3.8 \\
\hline 2007 & $5,511.88$ & 1.1 & 1.3 \\
\hline 2008 & $6,633.55$ & 1.1 & 7.9 \\
\hline 2009 & $6,584.54$ & 1.2 & 2.2 \\
\hline 2010 & $6,557.80$ & 1.0 & 2.3 \\
\hline 2011 & $7,289.72$ & 1.0 & 2.7 \\
\hline 2012 & $7,543.28$ & 1.0 & 6.8 \\
\hline 2013 & $8,307.23$ & 1.0 & 1.0 \\
\hline 2014 & $9,148.56$ & 1.0 & -1.1 \\
\hline
\end{tabular}

Source: Benin Republic National Statistics office
Table 4. Log of Regression Data for Benin Republic

\begin{tabular}{|c|c|c|c|}
\hline Year & GDP (\%) & $\begin{array}{c}\text { Unemployment } \\
\text { rate (\%) }\end{array}$ & $\begin{array}{c}\text { Inflation rate } \\
(\%)\end{array}$ \\
\hline 2001 & 3.40 & $(0.15)$ & 0.60 \\
\hline 2002 & 3.45 & $(0.15)$ & 0.40 \\
\hline 2003 & 3.55 & $(0.05)$ & 0.18 \\
\hline 2004 & 3.61 & 0.11 & $(0.05)$ \\
\hline 2005 & 3.64 & 0.11 & 0.73 \\
\hline 2006 & 3.67 & 0.04 & 0.58 \\
\hline 2007 & 3.74 & 0.04 & 0.11 \\
\hline 2008 & 3.82 & 0.04 & 0.90 \\
\hline 2009 & 3.82 & 0.08 & 0.34 \\
\hline 2010 & 3.82 & 0.00 & 0.36 \\
\hline 2011 & 3.86 & 0.00 & 0.43 \\
\hline 2012 & 3.88 & 0.00 & 0.83 \\
\hline 2013 & 3.92 & 0.00 & 0.00 \\
\hline 2014 & 3.96 & 0.00 & 0.00 \\
\hline
\end{tabular}

Source: Own Computation

$$
\mathrm{GDP}=\mathrm{F}\left(\mathrm{X}_{1}+\mathrm{X}_{2}\right)+\mathrm{U}_{\mathrm{t}}
$$

$$
\begin{array}{lll}
\text { GDP } & = & \text { Dependable }(\mathrm{Y}) \\
\text { UNEMPL } & = & \text { Independent }\left(\mathrm{X}_{1}\right) \\
\text { INFLA } & = & \text { Independent }\left(\mathrm{X}_{2}\right)
\end{array}
$$

The model above was built to ascertain the impact of unemployment and inflation on economic growth as well as effect of inflation variable on Unemployment.

$\mathrm{GDP}_{\mathrm{t}}=3.59+0.15 \mathrm{GDP}_{\mathrm{t}-1}-0.02$ INFLA $_{\mathrm{t}}-1.34 \mathrm{UNEMPL}_{\mathrm{t}-1}+\mathrm{U}_{\mathrm{t}}$

T-statistics are in parenthesized

$$
\mathrm{R}^{2}=0.856 \quad \text { Adjusted } \mathrm{R}^{2}=0.813
$$$$
\text { F-Statistics }=19.81 \quad \mathrm{D}-\mathrm{W}=2.11
$$

The above equation (10) shows that a unit increase on economic growth proxies by (GDP) would bring about -0.02 decrease in the prices of goods and services and -1.34 decreases in the unemployment rate.

$$
\begin{array}{r}
\mathrm{UNEMPLY}=0.003+0.52 \mathrm{UR}_{\mathrm{t}-1}+0.014 \mathrm{IR}+\mathrm{U}_{\mathrm{t}} \\
(0.163)(1.906)
\end{array}
$$

T-statistics are in parenthesized

$\mathrm{R}^{2}=0.351 \quad$ Adjusted $\mathrm{R}^{2}=-0.222$

F-Statistics $=2.7 \quad \mathrm{D}-\mathrm{W}=1.62$

The above equation (11)shows that a unit increase on unemployment rate would bring about -0.421 decrease in inflation rate in the short-run when there is tradeoff but in the long-run when tradeoff disappearance, a unit increase on unemployment rate 
3.9.3. Cape Verde econometric Model

Table 5. Regression Data for Cape Verde

\begin{tabular}{|c|c|c|c|}
\hline Year & $\begin{array}{c}\text { GDP (US } \\
\text { \$ Million) }\end{array}$ & $\begin{array}{c}\text { Unemployment } \\
\text { rate (\%) }\end{array}$ & $\begin{array}{c}\text { Inflation rate } \\
(\%)\end{array}$ \\
\hline 2001 & 640.31 & 9.7 & 3.3 \\
\hline 2002 & 706.21 & 9.7 & 1.9 \\
\hline 2003 & 925.69 & 9.6 & 1.2 \\
\hline 2004 & $1,051.19$ & 9.6 & -1.9 \\
\hline 2005 & $1,105.39$ & 9.6 & 0.4 \\
\hline 2006 & $1,259.96$ & 9.5 & 8.3 \\
\hline 2007 & $1,513.93$ & 9.4 & 8.3 \\
\hline 2008 & $1,789.33$ & 9.4 & 24.1 \\
\hline 2009 & $1,711.82$ & 9.4 & 11.0 \\
\hline 2010 & $1,664.31$ & 9.3 & 6.4 \\
\hline 2011 & $1,864.83$ & 9.2 & 4.5 \\
\hline 2012 & $1,756.23$ & 9.1 & 2.5 \\
\hline 2013 & $1,861.31$ & 9.0 & 1.5 \\
\hline 2014 & $1,972.69$ & 9.2 & -0.2 \\
\hline
\end{tabular}

Source: Cabo Verde National Statistics office

Table 6. Log of Regression Data for Cape Verde

\begin{tabular}{|c|c|c|c|}
\hline Year & GDP (\%) & $\begin{array}{c}\text { Unemployment } \\
\text { rate (\%) }\end{array}$ & $\begin{array}{c}\text { Inflation rate } \\
(\%)\end{array}$ \\
\hline 2001 & 2.81 & 0.99 & 0.52 \\
\hline 2002 & 2.85 & 0.99 & 0.28 \\
\hline 2003 & 2.97 & 0.98 & 0.08 \\
\hline 2004 & 3.02 & 0.98 & 0.00 \\
\hline 2005 & 3.04 & 0.98 & $(0.40)$ \\
\hline 2006 & 3.10 & 0.98 & 0.92 \\
\hline 2007 & 3.18 & 0.97 & 0.92 \\
\hline 2008 & 3.25 & 0.97 & 1.38 \\
\hline 2009 & 3.23 & 0.97 & 1.04 \\
\hline 2010 & 3.22 & 0.97 & 0.81 \\
\hline 2011 & 3.27 & 0.96 & 0.65 \\
\hline 2012 & 3.24 & 0.96 & 0.40 \\
\hline 2013 & 3.27 & 0.95 & 0.18 \\
\hline 2014 & 3.30 & 0.96 & 0.00 \\
\hline
\end{tabular}

Source: Own computation

$$
\text { GDP }=\mathrm{F}\left(\mathrm{X}_{1}+\mathrm{X}_{2}\right)+\mathrm{U}_{\mathrm{t}}
$$

$\begin{array}{lll}\text { GDP } & = & \text { Dependable }(\mathrm{Y}) \\ \text { UNEMPL } & = & \text { Independent }\left(\mathrm{X}_{1}\right) \\ \text { INFLA } & = & \text { Independent }\left(\mathrm{X}_{2}\right)\end{array}$

The model above was built to ascertain the impact of unemployment and inflation on economic growth as well as effect of inflation variable on Unemployment.

$$
\begin{aligned}
\operatorname{GDP}_{\mathrm{t}-1}= & 44.15+0.17 \text { INFLA }_{\mathrm{t}}-42.58 \mathrm{UNEMPL}_{\mathrm{t}-1}+\mathrm{U}_{\mathrm{t}} \\
& (2.46)(0.334) \quad(-2.308)
\end{aligned}
$$

$\mathrm{T}$-statistics are in parenthesized

$\mathrm{R}^{2}=0.856 \quad$ Adjusted $\mathrm{R}^{2}=0.813$

F-Statistics $=19.81 \quad \mathrm{D}-\mathrm{W}=2.11$

The above equation (13) shows that a unit increase on economic growth proxies by (GDP) would bring about 0.17 increases in the prices of goods and services which cause -42.58 decreases in the unemployment rate in the previous year.

$$
\mathrm{UR}=\mathrm{F}(\mathrm{X})+\mathrm{U}_{\mathrm{t}}
$$

$\mathrm{UNMPL}=$ Dependable $(\mathrm{Y})$

$\mathrm{INFLA}=$ Independent $(\mathrm{X})$

$$
\begin{gathered}
\mathrm{UR}_{\mathrm{t}-1}=0.532+0.318 \mathrm{UR}_{\mathrm{t}-2}-0.48 \mathrm{IR}_{\mathrm{t}}+0.588 \mathrm{IR}_{\mathrm{t}-1}+\mathrm{U}_{\mathrm{t}} \\
(3.745)(1.899) \quad(-2.196)
\end{gathered}
$$

$\mathrm{T}$-statistics are in parenthesized

$\mathrm{R}^{2}=0.676 \quad$ Adjusted $\mathrm{R}^{2}=-0.553$

F-Statistics $=5.55 \quad \mathrm{D}-\mathrm{W}=2.51$

The above equation (11)shows that a unit increase on unemployment rate would bring about -0.421 decrease in inflation rate in the short-run when there is tradeoff but in the long-run when tradeoff disappearance, a unit increase on unemployment rate

3.9.4. Cote d'ivoire econometric Model

Table 7. Regression Data for Cote d'ivoire

\begin{tabular}{|c|c|c|c|}
\hline Year & $\begin{array}{c}\text { GDP (US } \\
\text { \$ Million) }\end{array}$ & $\begin{array}{c}\text { Unemployment } \\
\text { rate (\%) }\end{array}$ & $\begin{array}{c}\text { Inflation rate } \\
(\%)\end{array}$ \\
\hline 2001 & $11,192.56$ & 4.1 & 4.3 \\
\hline 2002 & $12,346.92$ & 4.1 & 3.1 \\
\hline 2003 & $15,306.60$ & 4.1 & 3.3 \\
\hline 2004 & $16,554.44$ & 4.1 & 1.4 \\
\hline 2005 & $17,084.94$ & 4.1 & 3.9 \\
\hline 2006 & $17,275.64$ & 4.1 & 2.5 \\
\hline 2007 & $19,696.02$ & 4.1 & 1.9 \\
\hline 2008 & $23,280.88$ & 4.1 & 6.3 \\
\hline 2009 & $23,043.24$ & 4.1 & 1.0 \\
\hline 2010 & $22,920.87$ & 4.1 & 1.7 \\
\hline 2011 & $24,074.54$ & 4.1 & 4.9 \\
\hline 2012 & $24,680.37$ & 4.1 & 1.3 \\
\hline 2013 & $28,593.23$ & 4.1 & 2.6 \\
\hline 2014 & $33,126.43$ & 4.0 & 0.5 \\
\hline
\end{tabular}

Source: Cote d'ivoire National Statistics office 
Table 8. Log of Regression Data for Cote d'ivoire

\begin{tabular}{|c|c|c|c|}
\hline Year & GDP (\%) & $\begin{array}{c}\text { Unemployment } \\
\text { rate (\%) }\end{array}$ & $\begin{array}{c}\text { Inflation rate } \\
(\%)\end{array}$ \\
\hline 2001 & 4.05 & 0.61 & 0.63 \\
\hline 2002 & 4.09 & 0.61 & 0.49 \\
\hline 2003 & 4.18 & 0.61 & 0.52 \\
\hline 2004 & 4.22 & 0.61 & 0.15 \\
\hline 2005 & 4.23 & 0.61 & 0.59 \\
\hline 2006 & 4.24 & 0.61 & 0.40 \\
\hline 2007 & 4.29 & 0.61 & 0.28 \\
\hline 2008 & 4.37 & 0.61 & 0.80 \\
\hline 2009 & 4.36 & 0.61 & 0.00 \\
\hline 2010 & 4.36 & 0.61 & 0.23 \\
\hline 2011 & 4.38 & 0.61 & 0.69 \\
\hline 2012 & 4.39 & 0.61 & 0.11 \\
\hline 2013 & 4.46 & 0.61 & 0.41 \\
\hline 2014 & 4.52 & 0.60 & $(0.30)$ \\
\hline
\end{tabular}

Source: Own computation

The model above was built to ascertain the impact of unemployment and inflation on economic growth as well as effect of inflation variable on Unemployment.

$$
\begin{aligned}
\mathrm{GDP}_{\mathrm{t}-1} & =2.297-0.49 \text { INFLA }_{\mathrm{t}}+0.51 \text { INFLA }_{\mathrm{t}-1}+\mathrm{U}_{\mathrm{t}} \\
& (2.57) \quad(-0.44) \quad(3.023)
\end{aligned}
$$

T-statistics are in parenthesized

$$
\mathrm{R}^{2}=0.524 \quad \text { Adjusted } \mathrm{R}^{2}=0.428
$$

F-Statistics $=5.49 \mathrm{D}-\mathrm{W}=2.52$

The above equation (16) shows that a unit increase on economic growth proxies by (GDP) in the previous year causes prices of the goods and services to decrease by- 0.49 .

$$
\mathrm{UNMPL}=\mathrm{F}(\mathrm{X})+\mathrm{U}_{\mathrm{t}}
$$

UNMPL $=$ Dependable $(\mathrm{Y})$

INFLA $=$ Independent $(\mathrm{X})$

$$
\begin{aligned}
\mathrm{UR}_{\mathrm{t}-1} & =0.341+0.48 \mathrm{UR}_{\mathrm{t}-2}-0.07 \mathrm{IR}+\mathrm{U}_{\mathrm{t}} \\
& (2.547) \quad(2.706) \quad(-0.391)
\end{aligned}
$$

$\mathrm{T}$-statistics are in parenthesized

$$
\mathrm{R}^{2}=0.466 \quad \text { Adjusted } \mathrm{R}^{2}=-0.396
$$

$$
\text { F-Statistics }=4.37 \quad \mathrm{D}-\mathrm{W}=2.48
$$

The above equation (18)shows that a unit increase on unemployment rate would bring about -0.07 decrease in inflation rate in the short-run when there is tradeoff but in the long-run when tradeoff disappearance, a unit increase on unemployment rate

\subsubsection{The Gambian econometric Model}

Table 9. Regression Data for Gambia

\begin{tabular}{|c|c|c|c|}
\hline Year & $\begin{array}{c}\text { GDP (US } \\
\text { \$ Million) }\end{array}$ & $\begin{array}{c}\text { Unemployment } \\
\text { rate (\%) }\end{array}$ & $\begin{array}{c}\text { Inflation rate } \\
(\%)\end{array}$ \\
\hline 2001 & 687.41 & 7.2 & 2.1 \\
\hline 2002 & 578.24 & 7.3 & 0.0 \\
\hline 2003 & 487.04 & 7.1 & 2.2 \\
\hline 2004 & 578.79 & 7.1 & 0.4 \\
\hline 2005 & 624.17 & 7.2 & 3.7 \\
\hline 2006 & 655.07 & 7.1 & 2.1 \\
\hline 2007 & 798.87 & 7.1 & 5.4 \\
\hline 2008 & 965.77 & 7.1 & 4.4 \\
\hline 2009 & 900.64 & 7.0 & 4.6 \\
\hline 2010 & 951.83 & 7.0 & 5.0 \\
\hline 2011 & 904.26 & 7.2 & 4.8 \\
\hline 2012 & 914.10 & 7.0 & 4.3 \\
\hline 2013 & 901.89 & 7.0 & 5.7 \\
\hline 2014 & 914.54 & 7.0 & 5.9 \\
\hline
\end{tabular}

Source: Gambia National Statistical office

Table 10. Log of Regression Data for Gambia

\begin{tabular}{|c|c|c|c|}
\hline Year & GDP (\%) & $\begin{array}{c}\text { Unemployment } \\
\text { rate (\%) }\end{array}$ & $\begin{array}{c}\text { Inflation rate } \\
(\%)\end{array}$ \\
\hline 2001 & 2.84 & 0.86 & 0.32 \\
\hline 2002 & 2.76 & 0.86 & 0.00 \\
\hline 2003 & 2.69 & 0.85 & 0.34 \\
\hline 2004 & 2.76 & 0.85 & $(0.40)$ \\
\hline 2005 & 2.80 & 0.86 & 0.57 \\
\hline 2006 & 2.82 & 0.85 & 0.32 \\
\hline 2007 & 2.90 & 0.85 & 0.73 \\
\hline 2008 & 2.98 & 0.85 & 0.64 \\
\hline 2009 & 2.95 & 0.85 & 0.66 \\
\hline 2010 & 2.98 & 0.85 & 0.70 \\
\hline 2011 & 2.96 & 0.86 & 0.68 \\
\hline 2012 & 2.96 & 0.85 & 0.63 \\
\hline 2013 & 2.96 & 0.85 & 0.76 \\
\hline 2014 & 2.96 & 0.85 & 0.77 \\
\hline
\end{tabular}

Source: Own computation

The model above was built to ascertain the impact of unemployment and inflation on economic growth as well as effect of inflation variable on Unemployment.

$$
\begin{aligned}
\mathrm{GDP}_{\mathrm{t}-1}= & 2.177-1.958 \mathrm{INFLA}_{\mathrm{t}}+3.41 \mathrm{INFLA}_{\mathrm{t}-2}+\mathrm{U}_{\mathrm{t}} \\
& (3.84) \quad(-1.112)
\end{aligned}
$$

T-statistics are in parenthesized

$$
\mathrm{R}^{2}=0.448 \quad \text { Adjusted } \mathrm{R}^{2}=0.325
$$

F-Statistics $=3.65 \mathrm{D}-\mathrm{W}=2.15$

The above equation (19) shows that a unit increase on economic growth proxies by (GDP) in the previous year causes prices of the goods and services to decrease by- 0.49 . 


$$
\mathrm{UNMPL}=\mathrm{F}(\mathrm{X})+\mathrm{U}_{\mathrm{t}}
$$

UNMPL $=$ Dependable $(\mathrm{Y})$

INFLA $=$ Independent $(\mathrm{X})$

$$
\begin{aligned}
\mathrm{UR}= & 0.858+0.006 \mathrm{IR}+\mathrm{U}_{\mathrm{t}} \\
& (262.24)(1.742)
\end{aligned}
$$

$\mathrm{T}$-statistics are in parenthesized

$\mathrm{R}^{2}=0.216 \quad$ Adjusted $\mathrm{R}^{2}=-0.145$

$$
\text { F-Statistics }=3.04 \quad \mathrm{D}-\mathrm{W}=2.16
$$

The above equation (20) shows that a unit increase on unemployment rate would bring about -0.858 decrease in inflation rate in the short-run when there is tradeoff but in the long-run when tradeoff disappearance, a unit increase on unemployment rate

\subsubsection{Ghanaian econometric Model}

Table 11. Regression Data for Ghana

\begin{tabular}{|c|c|c|c|}
\hline Year & $\begin{array}{c}\text { GDP (US } \\
\text { \$ Million) }\end{array}$ & $\begin{array}{c}\text { Unemployment } \\
\text { rate (\%) }\end{array}$ & $\begin{array}{c}\text { Inflation rate } \\
(\%)\end{array}$ \\
\hline 2001 & $8,517.41$ & 10.0 & 32.9 \\
\hline 2002 & $9,881.71$ & 9.8 & 14.8 \\
\hline 2003 & $12,231.91$ & 8.1 & 26.7 \\
\hline 2004 & $14,233.02$ & 6.9 & 12.6 \\
\hline 2005 & $17,198.51$ & 3.8 & 15.1 \\
\hline 2006 & $20,410.33$ & 3.6 & 10.9 \\
\hline 2007 & $24,757.55$ & 2.8 & 10.7 \\
\hline 2008 & $28,528.01$ & 4.3 & 16.5 \\
\hline 2009 & $25,977.85$ & 2.2 & 19.3 \\
\hline 2010 & $32,174.21$ & 5.3 & 10.7 \\
\hline 2011 & $39,199.66$ & 4.6 & 8.7 \\
\hline 2012 & $41,740.93$ & 3.6 & 9.2 \\
\hline 2013 & $47,829.61$ & 1.8 & 11.6 \\
\hline 2014 & $54,806.44$ & 2.4 & 15.5 \\
\hline
\end{tabular}

Source: Ghana National Statistics office

Table 12. Log of Regression Data for Ghana

\begin{tabular}{|c|c|c|c|}
\hline Year & GDP (\%) & $\begin{array}{c}\text { Unemployment } \\
\text { rate (\%) }\end{array}$ & $\begin{array}{c}\text { Inflation rate } \\
(\%)\end{array}$ \\
\hline 2001 & 3.93 & 1.00 & 1.52 \\
\hline 2002 & 3.99 & 0.99 & 1.17 \\
\hline 2003 & 4.09 & 0.91 & 1.43 \\
\hline 2004 & 4.15 & 0.84 & 1.10 \\
\hline 2005 & 4.24 & 0.58 & 1.18 \\
\hline 2006 & 4.31 & 0.56 & 1.04 \\
\hline 2007 & 4.39 & 0.45 & 1.03 \\
\hline 2008 & 4.46 & 0.63 & 1.22 \\
\hline 2009 & 4.41 & 0.34 & 1.29 \\
\hline 2010 & 4.51 & 0.72 & 1.03 \\
\hline 2011 & 4.59 & 0.66 & 0.94 \\
\hline 2012 & 4.62 & 0.56 & 0.96 \\
\hline 2013 & 4.68 & 0.26 & 1.06 \\
\hline 2014 & 4.74 & 0.38 & 1.19 \\
\hline
\end{tabular}

Source: Own computation
The model above was built to ascertain the impact of unemployment and inflation on economic growth as well as effect of inflation variable on Unemployment.

$$
\begin{aligned}
\mathrm{GDP}_{\mathrm{t}-1}= & 5.00-2.76 \mathrm{INFLA}_{\mathrm{t}}+2.06 \mathrm{INFLA}_{\mathrm{t}-2}+\mathrm{U}_{\mathrm{t}} \\
& (0.557)
\end{aligned}
$$

$\mathrm{T}$-statistics are in parenthesized

$\mathrm{R}^{2}=0.752 \quad$ Adjusted $\mathrm{R}^{2}=0.706$

F-Statistics $=16.64 \quad \mathrm{D}-\mathrm{W}=1.54$

The above equation (21) shows that a unit increase on economic growth proxies by (GDP) in the previous year causes prices of the goods and services to decrease by-2.76.

$$
\mathrm{UNMPL}=\mathrm{F}(\mathrm{X})+\mathrm{U}_{\mathrm{t}}
$$

UNMPL $=$ Dependable $(\mathrm{Y})$

INFLA $=$ Independent $(\mathrm{X})$

$$
\begin{aligned}
\mathrm{UR}_{\mathrm{t}-1}= & -0.402+0.12 \mathrm{UR}_{\mathrm{t}-2}+0.263 \mathrm{IR}+0.593 \mathrm{IR}_{\mathrm{t}-1}+\mathrm{U}_{\mathrm{t}} \\
& (-0.674) \quad(0.577)(0.653) \quad(2.878)
\end{aligned}
$$

$\mathrm{T}$-statistics are in parenthesized

$$
\begin{array}{ll}
\mathrm{R}^{2}=0.541 & \text { Adjusted } \mathrm{R}^{2}=-0.404 \\
\text { F-Statistics }=3.94 & \mathrm{D}-\mathrm{W}=1.61
\end{array}
$$

The above equation (23) shows that a unit increase on unemployment rate would bring about 0.59 increase in inflation rate in the short-run when there is tradeoff but in the long-run when tradeoff disappearance, a unit increase on unemployment rate

\subsubsection{Guinea Bissau econometric Model}

Table 13. Regression Data for Guinea Bissau

\begin{tabular}{|c|c|c|c|}
\hline Year & $\begin{array}{c}\text { GDP (US } \\
\text { \$ Million) }\end{array}$ & $\begin{array}{c}\text { Unemployment } \\
\text { rate (\%) }\end{array}$ & $\begin{array}{c}\text { Inflation rate } \\
(\%)\end{array}$ \\
\hline 2001 & 394.24 & 6.8 & 3.3 \\
\hline 2002 & 418.07 & 6.9 & 3.3 \\
\hline 2003 & 468.44 & 6.9 & -3.5 \\
\hline 2004 & 524.17 & 6.9 & 0.9 \\
\hline 2005 & 586.79 & 6.8 & 3.3 \\
\hline 2006 & 591.83 & 6.9 & 2.0 \\
\hline 2007 & 695.61 & 6.8 & 4.6 \\
\hline 2008 & 864.11 & 6.8 & 10.5 \\
\hline 2009 & 826.97 & 6.8 & -1.7 \\
\hline 2010 & 845.03 & 6.8 & 2.5 \\
\hline 2011 & 974.35 & 6.8 & 5.0 \\
\hline 2012 & 908.07 & 6.9 & 2.1 \\
\hline 2013 & $1,036.35$ & 6.8 & 1.2 \\
\hline 2014 & $1,182.76$ & 6.9 & -1.5 \\
\hline
\end{tabular}

Source: Guinea Bissau National Statistics office 
Table 14. Log of Regression Data for Guinea Bissau

\begin{tabular}{|c|c|c|c|}
\hline Year & GDP (\%) & $\begin{array}{c}\text { Unemployment } \\
\text { rate (\%) }\end{array}$ & $\begin{array}{c}\text { Inflation rate } \\
(\%)\end{array}$ \\
\hline 2001 & 2.60 & 0.83 & 0.52 \\
\hline 2002 & 2.62 & 0.84 & 0.52 \\
\hline 2003 & 2.67 & 0.84 & 0.00 \\
\hline 2004 & 2.72 & 0.84 & $(0.05)$ \\
\hline 2005 & 2.77 & 0.83 & 0.52 \\
\hline 2006 & 2.77 & 0.84 & 0.30 \\
\hline 2007 & 2.84 & 0.83 & 0.66 \\
\hline 2008 & 2.94 & 0.83 & 1.02 \\
\hline 2009 & 2.92 & 0.83 & 0.00 \\
\hline 2010 & 2.93 & 0.83 & 0.40 \\
\hline 2011 & 2.99 & 0.83 & 0.70 \\
\hline 2012 & 2.96 & 0.84 & 0.32 \\
\hline 2013 & 3.02 & 0.83 & 0.08 \\
\hline 2014 & 3.07 & 0.84 & 0.00 \\
\hline
\end{tabular}

Source: Own computation

The model above was built to ascertain the impact of unemployment and inflation on economic growth as well as effect of inflation variable on Unemployment.

$$
\mathrm{GDP}_{\mathrm{t}-1}=1.73+0.9876 \mathrm{INFLA}_{\mathrm{t}-1}+1.23 \mathrm{INFLA}_{\mathrm{t}-2}+\mathrm{U}_{\mathrm{t}}
$$

$$
\text { (3.794) (1.427) (1.699) }
$$

$\mathrm{T}$-statistics are in parenthesized

$$
\mathrm{R}^{2}=0.752 \quad \text { Adjusted } \mathrm{R}^{2}=0.706
$$

F-Statistics $=16.64 \quad \mathrm{D}-\mathrm{W}=1.54$

The above equation (24) shows that a unit increase on economic growth proxies by (GDP) in the previous year causes prices of the goods and services to increase by0.987.

$$
\mathrm{UNMPL}=\mathrm{F}(\mathrm{X})+\mathrm{U}_{\mathrm{t}}
$$

$$
\begin{aligned}
\mathrm{UNMPL}= & \text { Dependable }(\mathrm{Y}) \\
\mathrm{INFLA}= & \text { Independent }(\mathrm{X}) \\
\mathrm{UR}_{\mathrm{t}-1}= & 0.417+0.501 \mathrm{UR}_{\mathrm{t}-2}-0.003 \mathrm{IR}+\mathrm{U}_{\mathrm{t}} \\
& (2.629)(2.878)-0.019)
\end{aligned}
$$

T-statistics are in parenthesized

$$
\mathrm{R}^{2}=0.462 \quad \text { Adjusted } \mathrm{R}^{2}=-0.354
$$$$
\text { F-Statistics }=4.30 \quad \mathrm{D}-\mathrm{W}=2.39
$$

The above equation (26) shows that a unit increase on unemployment rate would bring about -0.003 decrease in inflation rate in the short-run when there is tradeoff but in the long-run when tradeoff disappearance, a unit increase on unemployment rate

\subsubsection{Malian econometric Model}

Table 15. Regression Data for Mali

\begin{tabular}{|c|c|c|c|}
\hline Year & $\begin{array}{c}\text { GDP (US } \\
\text { \$ Million) }\end{array}$ & $\begin{array}{c}\text { Unemployment } \\
\text { rate }(\%)\end{array}$ & $\begin{array}{c}\text { Inflation rate } \\
(\%)\end{array}$ \\
\hline 2001 & $3,017.63$ & 8.3 & 5.2 \\
\hline 2002 & $3,189.06$ & 8.4 & 5.0 \\
\hline 2003 & $4,221.53$ & 8.6 & -1.3 \\
\hline 2004 & $4,982.27$ & 8.8 & -3.1 \\
\hline 2005 & $5,486.32$ & 8.5 & 6.4 \\
\hline 2006 & $6,122.65$ & 8.3 & 1.5 \\
\hline 2007 & $7,145.36$ & 8.5 & 1.4 \\
\hline 2008 & $8,737.66$ & 8.4 & 9.2 \\
\hline 2009 & $8,964.48$ & 8.5 & 2.5 \\
\hline 2010 & $9,400.18$ & 8.1 & 1.1 \\
\hline 2011 & $10,647.55$ & 8.1 & 2.9 \\
\hline 2012 & $10,340.80$ & 8.1 & 5.4 \\
\hline 2013 & $10,942.73$ & 8.1 & -0.6 \\
\hline 2014 & $11,579.69$ & 8.1 & 0.9 \\
\hline
\end{tabular}

Source: Mali National Statistics office

Table 16. Log of Regression Data for Mali

\begin{tabular}{|c|c|c|c|}
\hline Year & GDP (\%) & $\begin{array}{c}\text { Unemployment } \\
\text { rate (\%) }\end{array}$ & $\begin{array}{c}\text { Inflation rate } \\
(\%)\end{array}$ \\
\hline 2001 & 3.48 & 0.92 & 0.72 \\
\hline 2002 & 3.50 & 0.92 & 0.70 \\
\hline 2003 & 3.63 & 0.93 & 0.00 \\
\hline 2004 & 3.70 & 0.94 & 0.00 \\
\hline 2005 & 3.74 & 0.93 & 0.81 \\
\hline 2006 & 3.79 & 0.92 & 0.18 \\
\hline 2007 & 3.85 & 0.93 & 0.15 \\
\hline 2008 & 3.94 & 0.92 & 0.96 \\
\hline 2009 & 3.95 & 0.93 & 0.40 \\
\hline 2010 & 3.97 & 0.91 & 0.04 \\
\hline 2011 & 4.03 & 0.91 & 0.46 \\
\hline 2012 & 4.01 & 0.91 & 0.73 \\
\hline 2013 & 4.04 & 0.91 & 0.00 \\
\hline 2014 & 4.06 & 0.91 & $(0.05)$ \\
\hline
\end{tabular}

Source: Own computation

The model above was built to ascertain the impact of unemployment and inflation on economic growth as well as effect of inflation variable on Unemployment.

$$
\begin{aligned}
\mathrm{GDP}_{\mathrm{t}-1} & =1.72+0.565 \mathrm{GDP}_{\mathrm{t}-2}-0.045 \mathrm{INFLA}_{\mathrm{t}-1}+\mathrm{U}_{\mathrm{t}} \\
& (3.291)(3.429) \quad(-0.070)
\end{aligned}
$$

$\mathrm{T}$-statistics are in parenthesized

$\mathrm{R}^{2}=0.752 \quad$ Adjusted $\mathrm{R}^{2}=0.706$

$$
\text { F-Statistics }=16.64 \quad \mathrm{D}-\mathrm{W}=1.54
$$

The above equation (27) shows that a unit increase on economic growth proxies by (GDP) in the two lag years causes prices of the goods and services to decrease by- 0.045 . 


$$
\mathrm{UNMPL}=\mathrm{F}(\mathrm{X})+\mathrm{U}_{\mathrm{t}}
$$

UNMPL $=$ Dependable $(\mathrm{Y})$

INFLA $=$ Independent $(\mathrm{X})$

URt-1 = $0.462+0.499 \mathrm{UR}_{\mathrm{t}-2}-0.002 \mathrm{IR}+\mathrm{Ut}$

$$
\text { (2.372) (2.683) -0.016) }
$$

$\mathrm{T}$-statistics are in parenthesized

$$
\mathrm{R}^{2}=0.461 \quad \text { Adjusted } \mathrm{R}^{2}=-0.353
$$

F-Statistics $=4.27 \mathrm{D}-\mathrm{W}=2.50$

The above equation (29) shows that a unit increase on unemployment rate would bring about -0.002 decrease in inflation rate in the short-run when there is tradeoff but in the long-run when tradeoff disappearance, a unit increase on unemployment rate.

\subsubsection{Niger econometric Model}

Table 17. Regression Data for Niger

\begin{tabular}{|c|c|c|c|}
\hline Year & $\begin{array}{c}\text { GDP (US } \\
\text { \$ Million) }\end{array}$ & $\begin{array}{c}\text { Unemployment } \\
\text { rate }(\%)\end{array}$ & $\begin{array}{c}\text { Inflation rate } \\
(\%)\end{array}$ \\
\hline 2001 & $1,880.69$ & 5.0 & 4.0 \\
\hline 2002 & $2,144.64$ & 5.0 & 2.6 \\
\hline 2003 & $2,640.61$ & 5.0 & -1.6 \\
\hline 2004 & $2,897.01$ & 5.0 & 0.3 \\
\hline 2005 & $3,369.01$ & 5.1 & 7.8 \\
\hline 2006 & $3,646.73$ & 5.1 & 0.0 \\
\hline 2007 & $4,283.55$ & 5.1 & 0.1 \\
\hline 2008 & $5,403.36$ & 5.1 & 11.3 \\
\hline 2009 & $5,397.12$ & 5.1 & 0.6 \\
\hline 2010 & $5,718.59$ & 5.1 & 0.8 \\
\hline 2011 & $6,409.17$ & 5.1 & 2.9 \\
\hline 2012 & $6,687.73$ & 5.1 & 0.5 \\
\hline 2013 & $7,407.42$ & 5.1 & 2.3 \\
\hline 2014 & $8,204.55$ & 5.1 & -0.8 \\
\hline
\end{tabular}

Source: Niger National Statistics office

Table 18. Log of Regression Data for Niger

\begin{tabular}{|c|c|c|c|}
\hline Year & GDP (\%) & $\begin{array}{c}\text { Unemployment } \\
\text { rate (\%) }\end{array}$ & Inflation rate (\%) \\
\hline 2001 & 3.27 & 0.70 & 0.60 \\
\hline 2002 & 3.33 & 0.70 & 0.41 \\
\hline 2003 & 3.42 & 0.70 & 0.00 \\
\hline 2004 & 3.46 & 0.70 & $(0.52)$ \\
\hline 2005 & 3.53 & 0.71 & 0.89 \\
\hline 2006 & 3.56 & 0.71 & 0.00 \\
\hline 2007 & 3.63 & 0.71 & $(1.00)$ \\
\hline 2008 & 3.73 & 0.71 & 1.05 \\
\hline 2009 & 3.73 & 0.71 & $(0.22)$ \\
\hline 2010 & 3.76 & 0.71 & $(0.10)$ \\
\hline 2011 & 3.81 & 0.71 & 0.46 \\
\hline 2012 & 3.83 & 0.71 & $(0.30)$ \\
\hline 2013 & 3.87 & 0.71 & 0.36 \\
\hline 2014 & 3.91 & 0.71 & 0.00 \\
\hline
\end{tabular}

Source: Own computation
The model above was built to ascertain the impact of unemployment and inflation on economic growth as well as effect of inflation variable on Unemployment.

$$
\begin{aligned}
\operatorname{GDP}_{\mathrm{t}-1} & =2.59-3.32 \mathrm{INFLA}_{\mathrm{t}}+1.95 \operatorname{INFLA}_{\mathrm{t}-1}+\mathrm{U}_{\mathrm{t}} \\
& (2.075)(-1.528)
\end{aligned}
$$

$\mathrm{T}$-statistics are in parenthesized

$\mathrm{R}^{2}=0.752 \quad$ Adjusted $\mathrm{R}^{2}=0.706$

F-Statistics $=16.64 \quad \mathrm{D}-\mathrm{W}=1.54$

The above equation (30) shows that a unit increase on economic growth proxies by (GDP) in the two lag years causes prices of the goods and services to decrease by-0.3.32.

$$
\mathrm{UNMPL}=\mathrm{F}(\mathrm{X})+\mathrm{U}_{\mathrm{t}}
$$

UNMPL $=$ Dependable $(\mathrm{Y})$

INFLA $=$ Independent $(\mathrm{X})$

$$
\begin{gathered}
\mathrm{UR}_{\mathrm{t}-1}=0.360+0.500 \mathrm{UR}_{\mathrm{t}-2}-0.003 \mathrm{IR}+\mathrm{U}_{\mathrm{t}} \\
(3.214) \quad(3.085) \quad(-0.267)
\end{gathered}
$$

$\mathrm{T}$-statistics are in parenthesized

$\mathrm{R}^{2}=0.476 \quad$ Adjusted $\mathrm{R}^{2}=-0.380$

F-Statistics $=4.98 \mathrm{D}-\mathrm{W}=2.54$

\begin{tabular}{|c|c|c|c|}
\hline Year & $\begin{array}{l}\text { GDP (US } \\
\text { \$ Million) }\end{array}$ & $\begin{array}{c}\text { Unemployment } \\
\text { rate }(\%)\end{array}$ & $\begin{array}{c}\text { Inflation rate } \\
(\%)\end{array}$ \\
\hline 2001 & $4,877.60$ & 9.7 & 3.1 \\
\hline 2002 & $5,333.86$ & 5.7 & 2.2 \\
\hline 2003 & $6,859.59$ & 8.7 & 0.0 \\
\hline 2004 & $8,031.34$ & 9.1 & 0.5 \\
\hline 2005 & $8,707.82$ & 9.2 & 1.7 \\
\hline 2006 & $9,358.66$ & 10.0 & 2.1 \\
\hline 2007 & $11,284.60$ & 8.8 & 5.9 \\
\hline 2008 & $13,386.37$ & 9.4 & 5.8 \\
\hline 2009 & $12,777.62$ & 9.9 & -2.2 \\
\hline 2010 & $12,925.68$ & 9.1 & 1.2 \\
\hline 2011 & $14,372.11$ & 10.4 & 3.4 \\
\hline 2012 & $14,047.57$ & 10.3 & 1.4 \\
\hline 2013 & $15,151.74$ & 10.3 & 0.7 \\
\hline 2014 & $16,342.71$ & 10.0 & -1.1 \\
\hline
\end{tabular}

The above equation (33) shows that a unit increase on unemployment rate would bring about -0.003 decrease in inflation rate in the short-run when there is tradeoff but in the long-run when tradeoff disappearance, a unit increase on unemployment rate.

3.9.10. Senegal econometric Model

Table 19. Regression Data for Senegal

Source: Senegal National Statistics office 
Table 20. Log of Regression Data for Senegal

\begin{tabular}{|c|c|c|c|}
\hline Year & GDP (\%) & $\begin{array}{c}\text { Unemployment } \\
\text { rate }(\%)\end{array}$ & $\begin{array}{c}\text { Inflation rate } \\
(\%)\end{array}$ \\
\hline 2001 & 3.69 & 0.99 & 0.49 \\
\hline 2002 & 3.73 & 0.76 & 0.34 \\
\hline 2003 & 3.84 & 0.94 & 0.00 \\
\hline 2004 & 3.90 & 0.96 & $(0.30)$ \\
\hline 2005 & 3.94 & 0.96 & 0.23 \\
\hline 2006 & 3.97 & 1.00 & 0.32 \\
\hline 2007 & 4.05 & 0.94 & 0.77 \\
\hline 2008 & 4.13 & 0.97 & 0.76 \\
\hline 2009 & 4.11 & 1.00 & 0.00 \\
\hline 2010 & 4.11 & 0.96 & 0.08 \\
\hline 2011 & 4.16 & 1.02 & 0.53 \\
\hline 2012 & 4.15 & 1.01 & 0.15 \\
\hline 2013 & 4.18 & 1.01 & $(0.15)$ \\
\hline 2014 & 4.21 & 1.00 & 0.00 \\
\hline
\end{tabular}

Source: Own computation

The model above was built to ascertain the impact of unemployment and inflation on economic growth as well as effect of inflation variable on Unemployment.

$$
\begin{aligned}
\mathrm{GDP}_{\mathrm{t}-1} & =1.83+.55 \mathrm{GDP}_{\mathrm{t}-2}-0.0087 \text { INFLA }_{\mathrm{t}-1}+\mathrm{U}_{\mathrm{t}} \\
& (3.149)
\end{aligned}
$$

T-statistics are in parenthesized

$\mathrm{R}^{2}=0.547 \quad$ Adjusted $\mathrm{R}^{2}=0.456$

F-Statistics $=6.03 \mathrm{D}-\mathrm{W}=2.79$

The above equation (30) shows that a unit increase on economic growth proxies by (GDP) in the two lag years causes prices of the goods and services to decrease by- 0.0087 .

$$
\mathrm{UNMPL}=\mathrm{F}(\mathrm{X})+\mathrm{U}_{\mathrm{t}}
$$

UNMPL $=$ Dependable $(\mathrm{Y})$

INFLA $=$ Independent $(\mathrm{X})$

$$
\mathrm{UR}=0.974+0.193 \mathrm{UR}_{\mathrm{t}-1-1}-0.198 \mathrm{UR}_{\mathrm{t}-2}-0.006 \mathrm{IR}+\mathrm{U}_{\mathrm{t}}
$$

$$
\text { (21.281) (3.396) (-4.628) (-0.141) }
$$

$\mathrm{T}$-statistics are in parenthesized

$\mathrm{R}^{2}=0.734 \quad$ Adjusted $\mathrm{R}^{2}=-0.635$

F-Statistics $=7.37 \quad \mathrm{D}-\mathrm{W}=1.51$

The above equation (36) shows that a unit increase on unemployment rate would bring about -0.006 decrease in inflation rate in the short-run when there is tradeoff but in the long-run when tradeoff disappearance, a unit increase on unemployment rate.

\section{Result and Discussion}

The different value of the dependent and independent variables are shown below in appendix 1-10. It indicates the impact of inflation on unemployment in ten (10) selected Economic Community of West Africa (ECOWAS) member's states.

\subsection{Summary of the Regression Result Models}

Nigeria Model

$$
\begin{gathered}
\mathrm{GDP}_{\mathrm{t}-1}=2.93-1.58 \mathrm{INFLA}_{\mathrm{t}}+3.74 \mathrm{INFLA}_{\mathrm{t}-1}+\mathrm{U}_{\mathrm{t}} \\
(2.034)(1.6605)
\end{gathered}
$$

UNEMPLO $=1.055+0.523$ UNEMPLO $_{\mathrm{t}-1}-0.421 \mathrm{INFLA}_{\mathrm{t}-1}+\mathrm{U}_{\mathrm{t}}$

$$
(1.055) \quad(3.027) \quad(-2.262)
$$

Benin Republic Model

$$
\begin{gathered}
\mathrm{GDP}_{\mathrm{t}}=3.59+0.15 \mathrm{GDP}_{\mathrm{t}-1}-0.02 \text { INFLA }_{\mathrm{t}}-1.34 \mathrm{UNEMPL}_{\mathrm{t}-1}+\mathrm{U}_{\mathrm{t}} \\
(29.31)(8.25) \quad(-1.559)(-3.292) \\
\mathrm{UNEMPLO}=0.003+0.52 \mathrm{UR}_{\mathrm{t}-1}+0.014 \mathrm{IR}+\mathrm{U}_{\mathrm{t}} \\
(0.163) \quad(1.906) \quad(0.390)
\end{gathered}
$$

Cape Verde Model

$$
\begin{gathered}
\mathrm{GDP}_{\mathrm{t}-1}=44.15+0.17 \text { INFLA }_{\mathrm{t}}-42.58 \mathrm{UNEMPL}_{\mathrm{t}-1}+\mathrm{U}_{\mathrm{t}} \\
(2.46)(0.334) \quad(-2.308) \\
\text { UNEMPLO }_{\mathrm{t}-1}=0.532+0.318 \mathrm{UR}_{\mathrm{t}-2}-0.48 \mathrm{IR}_{\mathrm{t}}+0.588 \mathrm{IR}_{\mathrm{t}-1}+\mathrm{U}_{\mathrm{t}}
\end{gathered}
$$

$$
(3.745) \quad(1.899) \quad(-2.196)(2.4403)
$$

Cote d'ivoire Model

$$
\begin{aligned}
\mathrm{GDP}_{\mathrm{t}-1} & =2.297-0.49 \text { INFLA }_{\mathrm{t}}+0.51 \mathrm{INFLA}_{\mathrm{t}-1}+\mathrm{U}_{\mathrm{t}} \\
(2.57) \quad(-0.44) & (3.023)
\end{aligned}
$$

UNEMPLO $_{\mathrm{t}-1}=0.341+0.48 \mathrm{UR}_{\mathrm{t}-2}-0.07 \mathrm{IR}+\mathrm{U}_{\mathrm{t}} \quad \mathrm{M}(8)$

$$
\text { (2.547) (2.706) (-0.391) }
$$

The Gambia Model

$$
\begin{aligned}
\mathrm{GDP}_{\mathrm{t}-1}= & 2.177-1.958 \mathrm{INFLA}_{\mathrm{t}}+3.41 \text { INFLA }_{\mathrm{t}-2}+\mathrm{U}_{\mathrm{t}} \\
(3.84) & (-1.112) \quad(2.216) \\
\mathrm{UNEMPLO}= & 0.858+0.006 \mathrm{IR}+\mathrm{U}_{\mathrm{t}} \\
& (262.24)(1.742)
\end{aligned}
$$

The Ghana Model

$$
\begin{aligned}
\mathrm{GDP}_{\mathrm{t}-1}= & 5.00-2.76 \mathrm{INFLA}_{\mathrm{t}}+2.06 \mathrm{INFLA}_{\mathrm{t}-2}+\mathrm{U}_{\mathrm{t}} \\
& (0.557) \quad(0.653)
\end{aligned}
$$

$\mathrm{UNEMPLO}_{\mathrm{t}-1}=-0.402+0.12 \mathrm{UR}_{\mathrm{t}-2}+0.263 \mathrm{IR}+0.593 \mathrm{IR}_{\mathrm{t}-1}+\mathrm{U}_{\mathrm{t}}$

$$
\begin{array}{llll}
(-0.674) & (0.577) & (0.653) & (2.878)
\end{array}
$$

The Guinea Bissau Model

$$
\mathrm{GDP}_{\mathrm{t}-1}=1.73+0.9876 \mathrm{INFLA}_{\mathrm{t}-1}+1.23 \text { INFLA }_{\mathrm{t}-2}+\mathrm{U}_{\mathrm{t}}
$$

$$
\text { (3.794) (1.427) (1.699) }
$$


$\mathrm{UNEMPLO}_{\mathrm{t}-1}=0.417+0.501 \mathrm{UR}_{\mathrm{t}-2}-0.003 \mathrm{IR}+\mathrm{U}_{\mathrm{t}}$

(2.629) (2.878) (-0.019)

Mali Model

$$
\begin{aligned}
& \mathrm{GDP}_{\mathrm{t}-1}=1.72+0.565 \mathrm{GDP}_{\mathrm{t}-2}-0.045 \mathrm{INFLA}_{\mathrm{t}-1}+\mathrm{U}_{\mathrm{t}} \\
& \begin{array}{lll}
(3.291) & (3.429) \quad(-0.070)
\end{array} \\
& \mathrm{UNEMPLO}_{\mathrm{t}-1}=0.462+0.499 \mathrm{UR}_{\mathrm{t}-2}-0.002 \mathrm{IR}+\mathrm{U}_{\mathrm{t}} \\
& \begin{array}{lll}
(2.372) & (2.683) & (-0.016)
\end{array}
\end{aligned}
$$

Niger Model

$$
\begin{aligned}
& \mathrm{GDP}_{\mathrm{t}-1}=2.59-3.32 \mathrm{INFLA}_{\mathrm{t}}+1.95 \mathrm{INFLA}_{\mathrm{t}-1}+\mathrm{U}_{\mathrm{t}} \\
& \text { (2.075) (-1.528) (1.0299) } \\
& \text { UNEMPLO }_{\mathrm{t}-1}=0.360+0.500 \mathrm{UR}_{\mathrm{t}-2^{2}}-0.003 \mathrm{IR}+\mathrm{U}_{\mathrm{t}} \\
& \text { (3.214) (3.085) (-0.267) }
\end{aligned}
$$

Senegal Model

$$
\begin{aligned}
\mathrm{GDP}_{\mathrm{t}-1}= & 1.83+.55 \mathrm{GDP}_{\mathrm{t}-2}-0.0087 \mathrm{INFLA}_{\mathrm{t}-1}+\mathrm{U}_{\mathrm{t}} \\
& (3.149) \quad(3.112) \quad(-0.009) \\
\mathrm{UNEMPLO}= & 0.974+0.193 \mathrm{UR}_{\mathrm{t}-1}-0.198 \mathrm{UR}_{\mathrm{t}-2}-0.006 \mathrm{IR}+\mathrm{U}_{\mathrm{t}}
\end{aligned}
$$

$$
\begin{array}{llll}
(21.281) & \text { (3.396) (-4.628) (-0.141) }
\end{array}
$$

The above equations shows that a unit increase on unemployment rate would bring about certain level of increase in inflation rate in the short-run when there is tradeoff but in the long-run when tradeoff disappearance, a unit increase on unemployment rate would bring about certain level of decreases in inflation rate.

The result from the model above shows that $\mathrm{R}^{2}$ is 0.50 . This simply implies that at least 50 percent of the variation in the unemployment rate is discussed by the independent variables. The coefficient of inflation rate is positive and significant at the level of significance of $5 \%$. This implies that the increase in unemployment rate have negative effect on the growth of the economy.

\section{Test of goodness of fit $\left(\mathrm{R}^{2}\right)$}

The model showed a significant coefficient of determination $\left(\mathrm{R}^{2}\right)$ which was $\left(\mathrm{R}^{2}=0.50 \%\right)$. This indicates that $50 \%$ of the variation in unemployment rate which is the dependent variable was explained by inflation which is the dependent variable. 0.50 or $50 \%$ was not given sufficient detailed report about because it was not essential to the model above.

\section{F-statistics}

At the significant level of $5 \%$, the model therefore indicates that there was prominent relationship between the inflation and unemployed rate, thus $\mathrm{F}$-test $=\mathrm{T}$-cal (4.91) $>\mathrm{T}$-tab (3.14) this reconfirmed the value of $\mathrm{R}^{2}=50 \%$ which was significant. This is because the f-cal (4.91) $>\mathrm{f}$-tab (3.14) at 5\% level of significance.

\section{Summary and Conclusion}

This study emphasises on the impact of inflation on unemployment rate in the ten (10) selected member's states (2001-2014). The dependent and independent variables used were macroeconomic indicators which are inflation and unemployment.

The observation from this study showed that both unemployment and inflation have positive influence on economic growth. This further explains that unemployment does not affect the economic growth of the country, rather inflation significantly improve the activities of the economy through growth in the per capita income in the ten (10) selected member's states.

\section{Recommendations}

The implication of the result from this study for major policy mainly implies that concrete effort should be made by the policy makers to identify potential economic sectors that has linkages capacity in the ten (10) selected member's states economy to ensure a creation of more job opportunities and activities in the economy which will therefore lead to a reduction in unemployment and the prices of commodities in their economy's. This effort will thus improve and increase economic activities in the country at large.

Furthermore, emphasis should be made to curb the surging rate of unemployment by making dedicated efforts to put in place labour intensive method of production instead of concentrating on capital intensive methods which will eliminate jobs that can be done by individuals. Finally, there must be concrete efforts to ensure that our porous borders are well managed to forestall leakages which is very pivotal for the reduction of unemployment and inflation; thereby improving the level of local production. 


\section{Appendixes}

Appendix 1a: Nigeria's Regression Output of GDP $_{t-1}=2.93-1.58 I N F L A_{t}+3.74 I N F L A_{t-1}+U_{t}$

\begin{tabular}{|c|c|c|c|c|}
\hline \multicolumn{5}{|c|}{ Dependent Variable: $\mathrm{GDP}_{\mathrm{t}-1}$} \\
\hline \multicolumn{5}{|c|}{ Method: Least Squares } \\
\hline \multicolumn{5}{|c|}{ Date: $04 / 21 / 16 \quad$ Time: $14: 15$} \\
\hline \multicolumn{5}{|c|}{ Sample: 20012014} \\
\hline \multicolumn{5}{|c|}{ Included observations: 14} \\
\hline Variable & Coefficient & Std. Error & t-Statistic & Prob. \\
\hline INFLA & -1.579837 & 1.605860 & -0.983795 & 0.3464 \\
\hline INFLA $_{t-1}$ & 3.736313 & 0.717644 & 5.206364 & 0.0003 \\
\hline $\mathrm{C}$ & 2.929250 & 2.034490 & 1.439796 & 0.1778 \\
\hline R-squared & 0.754143 & \multicolumn{2}{|c|}{ Mean dependent var } & 4.970714 \\
\hline Adjusted R-squared & 0.709441 & \multicolumn{2}{|c|}{ S.D. dependent var } & 1.455100 \\
\hline S.E. of regression & 0.784350 & \multicolumn{2}{|c|}{ Akaike info criterion } & 2.539486 \\
\hline Sum squared resid & 6.767247 & \multicolumn{2}{|c|}{ Schwarz criterion } & 2.676426 \\
\hline Log likelihood & -14.77640 & \multicolumn{2}{|c|}{ F-statistic } & 16.87069 \\
\hline Durbin-Watson stat & 1.601703 & \multicolumn{2}{|c|}{ Prob(F-statistic) } & 0.000445 \\
\hline
\end{tabular}

Source: Eviews 7.0

Appendix 1b: Regression Output of UNEMPLO $=1.055+0.523$ UNEMPLO $_{t-1}-0.421$ INFLA $_{t-1}+U_{t}$

\begin{tabular}{|c|c|c|c|c|}
\hline \multicolumn{5}{|c|}{ Dependent Variable: $\mathrm{UR}_{\mathrm{t}-1}$} \\
\hline \multicolumn{5}{|c|}{ Method: Least Squares } \\
\hline \multicolumn{5}{|c|}{ Date: 02/29/16 Time: 14:01 } \\
\hline \multicolumn{5}{|c|}{ Sample: 20012014} \\
\hline \multicolumn{5}{|c|}{ Included observations: 14} \\
\hline Variable & Coefficient & Std. Error & t-Statistic & Prob. \\
\hline $\mathrm{UR}_{\mathrm{t}-2}$ & 0.536557 & 0.159645 & 3.360935 & 0.0064 \\
\hline IR & -0.389919 & 0.509503 & -0.765294 & 0.4602 \\
\hline $\mathrm{C}$ & 0.989337 & 0.615722 & 1.606791 & 0.1364 \\
\hline R-squared & 0.577686 & \multicolumn{2}{|c|}{ Mean dependent var } & 1.128571 \\
\hline Adjusted R-squared & 0.500902 & \multicolumn{2}{|c|}{ S.D. dependent var } & 0.348378 \\
\hline S.E. of regression & 0.246118 & \multicolumn{2}{|c|}{ Akaike info criterion } & 0.221397 \\
\hline Sum squared resid & 0.666314 & \multicolumn{2}{|c|}{ Schwarz criterion } & 0.358338 \\
\hline Log likelihood & 1.450218 & \multicolumn{2}{|c|}{ F-statistic } & 7.523496 \\
\hline Durbin-Watson stat & 2.226739 & \multicolumn{2}{|c|}{ Prob(F-statistic) } & 0.008730 \\
\hline
\end{tabular}

Source: Eviews 7.0 
Appendix 2a: Benin Republic's Regression Output of GDP $_{t}=3.59+0.15 G D P_{t-1}-0.02 I N F L A_{t}-1.34 U N E M P L_{t-1}+U_{t}$

\begin{tabular}{|c|c|c|c|c|}
\hline \multicolumn{5}{|c|}{ Dependent Variable: $\mathrm{GDP}_{\mathrm{t}}$} \\
\hline \multicolumn{5}{|c|}{ Method: Least Squares } \\
\hline \multicolumn{5}{|c|}{ Date: $04 / 21 / 16 \quad$ Time: $16: 15$} \\
\hline \multicolumn{5}{|c|}{ Sample: 20012014} \\
\hline \multicolumn{5}{|c|}{ Included observations: 11} \\
\hline \multicolumn{5}{|c|}{ Excluded observations: 3} \\
\hline Variable & Coefficient & Std. Error & t-Statistic & Prob. \\
\hline GDPT & 0.150310 & 0.018225 & 8.247625 & 0.0002 \\
\hline INFLA & -0.020186 & 0.044095 & -0.457790 & 0.6632 \\
\hline UNEMPL & -0.258212 & 0.165549 & -1.559732 & 0.1698 \\
\hline UNEMPLT & -1.338142 & 0.406473 & -3.292078 & 0.0166 \\
\hline $\mathrm{C}$ & 3.589207 & 0.122453 & 29.31101 & 0.0000 \\
\hline R-squared & 0.961526 & \multicolumn{2}{|c|}{ Mean dependent var } & 3.775455 \\
\hline Adjusted R-squared & 0.935877 & \multicolumn{2}{|c|}{ S.D. dependent var } & 0.158326 \\
\hline S.E. of regression & 0.040092 & \multicolumn{2}{|c|}{ Akaike info criterion } & -3.292317 \\
\hline Sum squared resid & 0.009644 & \multicolumn{2}{|c|}{ Schwarz criterion } & -3.111455 \\
\hline Log likelihood & 23.10774 & \multicolumn{2}{|c|}{ F-statistic } & 37.48775 \\
\hline Durbin-Watson stat & 1.817968 & \multicolumn{2}{|c|}{ Prob(F-statistic) } & 0.000221 \\
\hline
\end{tabular}

Source: Eviews 7.0

APPENDIX 2b: Regression Output of UNEMPLY=0.003+0.52UNEMPLY $t-1+0.014 I N F L A+U_{t}$

\begin{tabular}{|c|c|c|c|c|}
\hline \multicolumn{5}{|c|}{ Dependent Variable: UR } \\
\hline \multicolumn{4}{|c|}{ Date: $02 / 29 / 16$ Time: $14: 18$} \\
\hline \multicolumn{4}{|c|}{ Sample(adjusted): 2005 2014 } \\
\hline \multicolumn{4}{|c|}{ Included observations: 10 after adjusting endpoints } \\
\hline Variable & Coefficient & Std. Error & t-Statistic & Prob. \\
\hline UR & 0.518376 & 0.271962 & 1.906059 & 0.0983 \\
\hline IR & 0.014290 & 0.036638 & 0.390031 & 0.7081 \\
\hline C & 0.003112 & 0.019048 & 0.163375 & 0.8748 \\
\hline R-squared & 0.414214 & Mean dependent var & 0.031000 \\
\hline Adjusted R-squared & 0.246846 & S.D. dependent var & 0.039001 \\
\hline S.E. of regression & 0.033847 & Akaike info criterion & -3.690598 \\
\hline Sum squared resid & 0.008019 & Schwarz criterion & -3.599822 \\
\hline Log likelihood & 21.45299 & F-statistic & 2.474874 \\
\hline Durbin-Watson stat & 2.406398 & Prob(F-statistic) & 0.153846 \\
\hline
\end{tabular}

Source: Eviews 7.0 
Appendix 3a: Cape Verde's Regression Output of GDP $_{t-1}=44.15+0.17$ INFLA $_{t}-42.58$ UNEMPL $_{t-1}+U_{t}$

\begin{tabular}{|c|c|c|c|c|}
\hline \multicolumn{5}{|c|}{ Dependent Variable: $\mathrm{GDP}_{\mathrm{t}-1}$} \\
\hline \multicolumn{5}{|c|}{ Method: Least Squares } \\
\hline \multicolumn{5}{|c|}{ Date: 04/21/16 Time: 16:39 } \\
\hline \multicolumn{5}{|c|}{ Sample: 20012014} \\
\hline \multicolumn{5}{|c|}{ Included observations: 13} \\
\hline \multicolumn{5}{|c|}{ Excluded observations: 1} \\
\hline Variable & Coefficient & Std. Error & t-Statistic & Prob. \\
\hline INFLA & 0.169262 & 0.507280 & 0.333667 & 0.7455 \\
\hline UNEMPL & -42.57734 & 18.44635 & -2.308172 & 0.0436 \\
\hline $\mathrm{C}$ & 44.15127 & 17.92110 & 2.463647 & 0.0335 \\
\hline R-squared & 0.351471 & \multicolumn{2}{|c|}{ Mean dependent var } & 2.879231 \\
\hline Adjusted R-squared & 0.221765 & \multicolumn{2}{|c|}{ S.D. dependent var } & 0.879445 \\
\hline S.E. of regression & 0.775826 & \multicolumn{2}{|c|}{ Akaike info criterion } & 2.529397 \\
\hline Sum squared resid & 6.019061 & \multicolumn{2}{|c|}{ Schwarz criterion } & 2.659770 \\
\hline Log likelihood & -13.44108 & \multicolumn{2}{|c|}{ F-statistic } & 2.709751 \\
\hline Durbin-Watson stat & 1.620809 & \multicolumn{2}{|c|}{ Prob(F-statistic) } & 0.114722 \\
\hline
\end{tabular}

Appendix 3b: Cape Verde's Regression Output ofUNEMPL $L_{t-1}=0.532+0.318 U R_{t-2}-0.48 I R_{t}+0.588 I R_{t-1}+U_{t}$

\begin{tabular}{|c|c|c|c|c|}
\hline \multicolumn{5}{|c|}{ Dependent Variable: $\mathrm{UR}_{\mathrm{t}-1}$} \\
\hline \multicolumn{5}{|c|}{ Method: Least Squares } \\
\hline \multicolumn{5}{|c|}{ Date: 02/29/16 Time: 14:27 } \\
\hline \multicolumn{5}{|c|}{ Sample: 20012014} \\
\hline \multicolumn{5}{|c|}{ Included observations: 12} \\
\hline \multicolumn{5}{|c|}{ Excluded observations: 2} \\
\hline Variable & Coefficient & Std. Error & t-Statistic & Prob. \\
\hline $\mathrm{UR}_{\mathrm{t}-2}$ & 0.318290 & 0.167524 & 1.899963 & 0.0940 \\
\hline IR & -0.482480 & 0.219645 & -2.196637 & 0.0593 \\
\hline $\mathrm{IR}_{\mathrm{t}-1}$ & 0.587689 & 0.244556 & 2.403085 & 0.0430 \\
\hline $\mathrm{C}$ & 0.532548 & 0.142220 & 3.744546 & 0.0057 \\
\hline R-squared & 0.675587 & \multicolumn{2}{|c|}{ Mean dependent var } & 0.890833 \\
\hline Adjusted R-squared & 0.553933 & \multicolumn{2}{|c|}{ S.D. dependent var } & 0.280793 \\
\hline S.E. of regression & 0.187537 & \multicolumn{2}{|c|}{ Akaike info criterion } & -0.248482 \\
\hline Sum squared resid & 0.281360 & \multicolumn{2}{|c|}{ Schwarz criterion } & -0.086846 \\
\hline Log likelihood & 5.490891 & \multicolumn{2}{|c|}{ F-statistic } & 5.553317 \\
\hline Durbin-Watson stat & 2.511287 & \multicolumn{2}{|c|}{ Prob(F-statistic) } & 0.023441 \\
\hline
\end{tabular}

Source: E-Views 7.0 
Appendix 4a: Cote d'ivoire's Regression Output of GDP t-1 $_{1}=2.297-0.49 \operatorname{INFLA}_{t}+0.51$ INFLA $_{t-1}+U_{t}$

\begin{tabular}{|c|c|c|c|c|}
\hline \multicolumn{5}{|c|}{ Dependent Variable: $\mathrm{GDP}_{\mathrm{t}-1}$} \\
\hline \multicolumn{5}{|c|}{ Method: Least Squares } \\
\hline \multicolumn{5}{|c|}{ Date: 04/22/16 Time: 09:18 } \\
\hline \multicolumn{5}{|c|}{ Sample(adjusted): 20012013} \\
\hline \multicolumn{5}{|c|}{ Included observations: 13 after adjusting endpoints } \\
\hline Variable & Coefficient & Std. Error & t-Statistic & Prob. \\
\hline INFLA $_{t}$ & -0.491325 & 1.116083 & -0.440223 & 0.6691 \\
\hline IMFLA $_{t-2}$ & 0.511027 & 0.169048 & 3.022965 & 0.0128 \\
\hline $\mathrm{C}$ & 2.297175 & 0.892749 & 2.573146 & 0.0277 \\
\hline R-squared & 0.523539 & \multicolumn{2}{|c|}{ Mean dependent var } & 3.935385 \\
\hline Adjusted R-squared & 0.428246 & \multicolumn{2}{|c|}{ S.D. dependent var } & 1.187586 \\
\hline S.E. of regression & 0.897986 & \multicolumn{2}{|c|}{ Akaike info criterion } & 2.821850 \\
\hline Sum squared resid & 8.063788 & \multicolumn{2}{|c|}{ Schwarz criterion } & 2.952223 \\
\hline Log likelihood & -15.34202 & \multicolumn{2}{|c|}{ F-statistic } & 5.494027 \\
\hline Durbin-Watson stat & 2.521703 & \multicolumn{2}{|c|}{ Prob(F-statistic) } & 0.024555 \\
\hline
\end{tabular}

Source: E-Views 7.0

Appendix 4b: Cote d'ivoire's Regression Output of $U_{N E M P L} L_{t-1}=0.341+0.48 U R_{t-2}-0.07 I R+U_{t}$

\begin{tabular}{|c|c|c|c|c|}
\hline \multicolumn{5}{|c|}{ Dependent Variable: $\mathrm{UR}_{\mathrm{t}-1}$} \\
\hline \multicolumn{5}{|c|}{ Method: Least Squares } \\
\hline \multicolumn{5}{|c|}{ Date: $02 / 29 / 16$ Time: $14: 36$} \\
\hline \multicolumn{5}{|c|}{ Sample(adjusted): 20012013} \\
\hline \multicolumn{5}{|c|}{ Included observations: 13 after adjusting endpoints } \\
\hline Variable & Coefficient & Std. Error & t-Statistic & Prob. \\
\hline $\mathrm{UR}_{\mathrm{t}-2}$ & 0.480615 & 0.177637 & 2.705611 & 0.0221 \\
\hline IR & -0.065692 & 0.167903 & -0.391251 & 0.7038 \\
\hline $\mathrm{C}$ & 0.341788 & 0.134176 & 2.547315 & 0.0290 \\
\hline R-squared & 0.466500 & \multicolumn{2}{|c|}{ Mean dependent var } & 0.563077 \\
\hline Adjusted R-squared & 0.359800 & \multicolumn{2}{|c|}{ S.D. dependent var } & 0.169184 \\
\hline S.E. of regression & 0.135368 & \multicolumn{2}{|c|}{ Akaike info criterion } & -0.962465 \\
\hline Sum squared resid & 0.183245 & \multicolumn{2}{|c|}{ Schwarz criterion } & -0.832092 \\
\hline Log likelihood & 9.256025 & \multicolumn{2}{|c|}{ F-statistic } & 4.372071 \\
\hline Durbin-Watson stat & 2.479299 & \multicolumn{2}{|c|}{ Prob(F-statistic) } & 0.043219 \\
\hline
\end{tabular}

Source: E-Views 7.0 
Appendix 5a: Gambia's Regression Output of GDP $_{t-1}=2.177-1.958$ INFLA $_{t}+3.41$ INFLA $_{t-2}+U_{t}$

\begin{tabular}{|c|c|c|c|c|}
\hline \multicolumn{5}{|c|}{ Dependent Variable: $\mathrm{GDP}_{\mathrm{t}-1}$} \\
\hline \multicolumn{5}{|c|}{ Method: Least Squares } \\
\hline \multicolumn{5}{|c|}{ Date: 04/22/16 Time: 09:53 } \\
\hline \multicolumn{5}{|c|}{ Sample: 20012014} \\
\hline \multicolumn{5}{|c|}{ Included observations: 12} \\
\hline \multicolumn{5}{|c|}{ Excluded observations: 2} \\
\hline Variable & Coefficient & Std. Error & t-Statistic & Prob. \\
\hline INFLA & -1.958112 & 1.759983 & -1.112575 & 0.2947 \\
\hline INFLAT2 & 3.409917 & 1.538594 & 2.216256 & 0.0539 \\
\hline $\mathrm{C}$ & 2.176977 & 0.565543 & 3.849359 & 0.0039 \\
\hline R-squared & 0.447979 & \multicolumn{2}{|c|}{ Mean dependent var } & 2.655833 \\
\hline Adjusted R-squared & 0.325307 & \multicolumn{2}{|c|}{ S.D. dependent var } & 0.840449 \\
\hline S.E. of regression & 0.690342 & \multicolumn{2}{|c|}{ Akaike info criterion } & 2.309058 \\
\hline Sum squared resid & 4.289145 & \multicolumn{2}{|c|}{ Schwarz criterion } & 2.430284 \\
\hline Log likelihood & -10.85435 & \multicolumn{2}{|c|}{ F-statistic } & 3.651861 \\
\hline Durbin-Watson stat & 2.154064 & \multicolumn{2}{|c|}{$\operatorname{Prob}(F$-statistic) } & 0.068992 \\
\hline
\end{tabular}

Source: E-Views 7.0

Appendix 5b: Gambia's Regression Output of $U_{t-1}=-0.402+0.12 U_{t-2}+0.263 I R+0.593 I R_{t-1}+U_{t}$

\begin{tabular}{|c|c|c|c|c|}
\hline \multicolumn{5}{|c|}{ Dependent Variable: UR } \\
\hline \multicolumn{5}{|c|}{ Method: Least Squares } \\
\hline \multicolumn{5}{|c|}{ Date: 02/29/16 Time: 14:37 } \\
\hline \multicolumn{5}{|c|}{ Sample: 20012014} \\
\hline \multicolumn{5}{|c|}{ Included observations: 13} \\
\hline \multicolumn{5}{|c|}{ Excluded observations: 1} \\
\hline Variable & Coefficient & Std. Error & t-Statistic & Prob. \\
\hline IR & 0.009645 & 0.005537 & 1.741916 & 0.1094 \\
\hline $\mathrm{C}$ & 0.858359 & 0.003273 & 262.2368 & 0.0000 \\
\hline R-squared & 0.216204 & \multicolumn{2}{|c|}{ Mean dependent var } & 0.853077 \\
\hline Adjusted R-squared & 0.144950 & \multicolumn{2}{|c|}{ S.D. dependent var } & 0.004804 \\
\hline S.E. of regression & 0.004442 & \multicolumn{2}{|c|}{ Akaike info criterion } & -7.854758 \\
\hline Sum squared resid & 0.000217 & \multicolumn{2}{|c|}{ Schwarz criterion } & -7.767842 \\
\hline Log likelihood & 53.05593 & \multicolumn{2}{|c|}{ F-statistic } & 3.034270 \\
\hline Durbin-Watson stat & 2.160899 & \multicolumn{2}{|c|}{ Prob(F-statistic) } & 0.109376 \\
\hline
\end{tabular}

Source: E-Views 7.0 
Appendix 6a: Ghana's Regression Output of GDP $_{t-1}=5.00-2.76$ INFLA $_{t}+2.06 I N F L A_{t-2}+U_{t}$

\begin{tabular}{|c|c|c|c|c|}
\hline \multicolumn{5}{|c|}{ Dependent Variable: $\mathrm{GDP}_{\mathrm{t}-1}$} \\
\hline \multicolumn{5}{|c|}{ Method: Least Squares } \\
\hline \multicolumn{5}{|c|}{ Date: $04 / 22 / 16 \quad$ Time: $11: 28$} \\
\hline \multicolumn{5}{|c|}{ Sample: 20012014} \\
\hline \multicolumn{5}{|c|}{ Included observations: 14} \\
\hline Variable & Coefficient & Std. Error & t-Statistic & Prob. \\
\hline INFLA & -2.761175 & 1.173761 & -2.352417 & 0.0383 \\
\hline INFLAT & 2.061411 & 0.566870 & 3.636479 & 0.0039 \\
\hline $\mathrm{C}$ & 5.009376 & 1.724680 & 2.904525 & 0.0143 \\
\hline R-squared & 0.751608 & \multicolumn{2}{|c|}{ Mean dependent var } & 4.026429 \\
\hline Adjusted R-squared & 0.706446 & \multicolumn{2}{|c|}{ S.D. dependent var } & 1.182121 \\
\hline S.E. of regression & 0.640481 & \multicolumn{2}{|c|}{ Akaike info criterion } & 2.134214 \\
\hline Sum squared resid & 4.512371 & \multicolumn{2}{|c|}{ Schwarz criterion } & 2.271155 \\
\hline Log likelihood & -11.93950 & \multicolumn{2}{|c|}{ F-statistic } & 16.64241 \\
\hline Durbin-Watson stat & 1.539361 & \multicolumn{2}{|c|}{ Prob(F-statistic) } & 0.000471 \\
\hline
\end{tabular}

Source: E-Views 7.0

Appendix 6b: Ghana's Regression Output of $U R_{t-1}=-0.402+0.12 U R_{t-2}+0.263 I R+0.593 I R_{t-1}+U_{t}$

\begin{tabular}{|c|c|c|c|c|}
\hline \multicolumn{5}{|c|}{ Dependent Variable: $\mathrm{UR}_{\mathrm{t}-1}$} \\
\hline \multicolumn{5}{|c|}{ Method: Least Squares } \\
\hline \multicolumn{5}{|c|}{ Date: $02 / 29 / 16 \quad$ Time: $14: 49$} \\
\hline \multicolumn{5}{|c|}{ Sample: 20012014} \\
\hline \multicolumn{5}{|c|}{ Included observations: 14} \\
\hline Variable & Coefficient & Std. Error & t-Statistic & Prob. \\
\hline $\mathrm{UR}_{\mathrm{t}-2}$ & 0.121209 & 0.209947 & 0.577329 & 0.5765 \\
\hline IR & 0.263392 & 0.403360 & 0.652996 & 0.5285 \\
\hline $\mathrm{IR}_{\mathrm{t}-1}$ & 0.593075 & 0.206061 & 2.878150 & 0.0164 \\
\hline $\mathrm{C}$ & -0.402393 & 0.596846 & -0.674200 & 0.5155 \\
\hline R-squared & 0.541819 & \multicolumn{2}{|c|}{ Mean dependent var } & 0.607143 \\
\hline Adjusted R-squared & 0.404365 & \multicolumn{2}{|c|}{ S.D. dependent var } & 0.284644 \\
\hline S.E. of regression & 0.219680 & \multicolumn{2}{|c|}{ Akaike info criterion } & 0.041671 \\
\hline Sum squared resid & 0.482595 & \multicolumn{2}{|c|}{ Schwarz criterion } & 0.224259 \\
\hline Log likelihood & 3.708301 & \multicolumn{2}{|c|}{ F-statistic } & 3.941816 \\
\hline Durbin-Watson stat & 1.605275 & \multicolumn{2}{|c|}{$\operatorname{Prob}($ F-statistic) } & 0.042921 \\
\hline
\end{tabular}

Source: E-Views 7.0 
Appendix 7a: Guinea Bissau's Regression Output of GDP $_{t-1}=1.73+0.9876$ INFLA $_{t-1}+1.23 I_{N F L A} A_{t-2}+U_{t}$

\begin{tabular}{|c|c|c|c|c|}
\hline \multicolumn{5}{|c|}{ Dependent Variable: $\mathrm{GDP}_{\mathrm{t}-1}$} \\
\hline \multicolumn{5}{|c|}{ Method: Least Squares } \\
\hline \multicolumn{5}{|c|}{ Date: 04/22/16 Time: 12:00 } \\
\hline \multicolumn{5}{|c|}{ Sample: 20012014} \\
\hline \multicolumn{5}{|c|}{ Included observations: 12} \\
\hline \multicolumn{5}{|c|}{ Excluded observations: 2} \\
\hline Variable & Coefficient & Std. Error & t-Statistic & Prob. \\
\hline INFLA $_{t-1}$ & 0.986934 & 0.691444 & 1.427353 & 0.1872 \\
\hline INFLA $_{t-2}$ & 1.219241 & 0.717719 & 1.698772 & 0.1236 \\
\hline $\mathrm{C}$ & 1.729302 & 0.455824 & 3.793791 & 0.0043 \\
\hline R-squared & 0.345541 & \multicolumn{2}{|c|}{ Mean dependent var } & 2.605000 \\
\hline Adjusted R-squared & 0.200105 & \multicolumn{2}{|c|}{ S.D. dependent var } & 0.833203 \\
\hline S.E. of regression & 0.745190 & \multicolumn{2}{|c|}{ Akaike info criterion } & 2.461964 \\
\hline Sum squared resid & 4.997780 & \multicolumn{2}{|c|}{ Schwarz criterion } & 2.583191 \\
\hline Log likelihood & -11.77179 & \multicolumn{2}{|c|}{ F-statistic } & 2.375903 \\
\hline Durbin-Watson stat & 1.674406 & \multicolumn{2}{|c|}{ Prob(F-statistic) } & 0.148413 \\
\hline
\end{tabular}

Source: E-Views 7.0

Appendix 7b: Guinea Bissau's Regression Output of $U_{t-1}=0.417+0.501 U^{-2-2}-003 I R+U_{t}$

\begin{tabular}{|c|c|c|c|c|}
\hline \multicolumn{5}{|c|}{ Dependent Variable: $\mathrm{UR}_{\mathrm{t}-1}$} \\
\hline \multicolumn{5}{|c|}{ Method: Least Squares } \\
\hline \multicolumn{5}{|c|}{ Date: $02 / 29 / 16 \quad$ Time: $14: 54$} \\
\hline \multicolumn{5}{|c|}{ Sample: 20012014} \\
\hline \multicolumn{5}{|c|}{ Included observations: 13} \\
\hline \multicolumn{5}{|c|}{ Excluded observations: 1} \\
\hline Variable & Coefficient & Std. Error & t-Statistic & Prob. \\
\hline $\mathrm{UR}_{\mathrm{t}-2}$ & 0.501402 & 0.174174 & 2.878741 & 0.0164 \\
\hline IR & -0.003426 & 0.173753 & -0.019719 & 0.9847 \\
\hline $\mathrm{C}$ & 0.416878 & 0.159145 & 2.619489 & 0.0256 \\
\hline R-squared & 0.462334 & \multicolumn{2}{|c|}{ Mean dependent var } & 0.769231 \\
\hline Adjusted R-squared & 0.354801 & \multicolumn{2}{|c|}{ S.D. dependent var } & 0.231173 \\
\hline S.E. of regression & 0.185688 & \multicolumn{2}{|c|}{ Akaike info criterion } & -0.330322 \\
\hline Sum squared resid & 0.344801 & \multicolumn{2}{|c|}{ Schwarz criterion } & -0.199949 \\
\hline Log likelihood & 5.147092 & \multicolumn{2}{|c|}{ F-statistic } & 4.299457 \\
\hline Durbin-Watson stat & 2.390147 & \multicolumn{2}{|c|}{ Prob(F-statistic) } & 0.044933 \\
\hline
\end{tabular}

Source: E-Views 7.0 
Appendix 8a: Mali's Regression Output of GDP $_{t-1}=1.72+0.565 G D P_{t-2}-0.045 I N F L A_{t-1}+U_{t}$

\begin{tabular}{|c|c|c|c|c|}
\hline \multicolumn{5}{|c|}{ Dependent Variable: $\mathrm{GDP}_{\mathrm{t}-1}$} \\
\hline \multicolumn{5}{|c|}{ Method: Least Squares } \\
\hline \multicolumn{5}{|c|}{ Date: 04/22/16 Time: 12:26 } \\
\hline \multicolumn{5}{|c|}{ Sample: 20012014} \\
\hline \multicolumn{5}{|c|}{ Included observations: 14} \\
\hline Variable & Coefficient & Std. Error & t-Statistic & Prob. \\
\hline $\mathrm{GDP}_{\mathrm{t}-2}$ & 0.564735 & 0.164677 & 3.429354 & 0.0056 \\
\hline INFLA $_{t-1}$ & -0.045246 & 0.642397 & -0.070434 & 0.9451 \\
\hline $\mathrm{C}$ & 1.722626 & 0.523398 & 3.291233 & 0.0072 \\
\hline R-squared & 0.563301 & \multicolumn{2}{|c|}{ Mean dependent var } & 3.545000 \\
\hline Adjusted R-squared & 0.483901 & \multicolumn{2}{|c|}{ S.D. dependent var } & 1.037480 \\
\hline S.E. of regression & 0.745326 & \multicolumn{2}{|c|}{ Akaike info criterion } & 2.437420 \\
\hline Sum squared resid & 6.110622 & \multicolumn{2}{|c|}{ Schwarz criterion } & 2.574361 \\
\hline Log likelihood & -14.06194 & \multicolumn{2}{|c|}{ F-statistic } & 7.094483 \\
\hline Durbin-Watson stat & 2.585058 & \multicolumn{2}{|c|}{$\operatorname{Prob}(F-$-statistic) } & 0.010495 \\
\hline
\end{tabular}

Source: E-Views 7.0

Appendix 8b: Mali's Regression Output of UNEMPL $L_{t-1}=0.462+0.499 U R_{t-2}-0.002 I R+U_{t}$

\begin{tabular}{|c|c|c|c|c|}
\hline \multicolumn{5}{|c|}{ Dependent Variable: $\mathrm{UR}_{\mathrm{t}-1}$} \\
\hline \multicolumn{5}{|c|}{ Method: Least Squares } \\
\hline \multicolumn{5}{|c|}{ Date: $02 / 29 / 16 \quad$ Time: $14: 59$} \\
\hline \multicolumn{5}{|c|}{ Sample(adjusted): 20012013} \\
\hline \multicolumn{5}{|c|}{ Included observations: 13 after adjusting endpoints } \\
\hline Variable & Coefficient & Std. Error & t-Statistic & Prob. \\
\hline $\mathrm{UR}_{\mathrm{t}-2}$ & 0.499778 & 0.186261 & 2.683220 & 0.0230 \\
\hline IR & -0.002947 & 0.182367 & -0.016157 & 0.9874 \\
\hline $\mathrm{C}$ & 0.462110 & 0.194739 & 2.372966 & 0.0391 \\
\hline R-squared & 0.460912 & \multicolumn{2}{|c|}{ Mean dependent var } & 0.851538 \\
\hline Adjusted R-squared & 0.353095 & \multicolumn{2}{|c|}{ S.D. dependent var } & 0.256022 \\
\hline S.E. of regression & 0.205920 & \multicolumn{2}{|c|}{ Akaike info criterion } & -0.123485 \\
\hline Sum squared resid & 0.424030 & \multicolumn{2}{|c|}{ Schwarz criterion } & 0.006888 \\
\hline Log likelihood & 3.802653 & \multicolumn{2}{|c|}{ F-statistic } & 4.274926 \\
\hline Durbin-Watson stat & 2.503428 & \multicolumn{2}{|c|}{ Prob(F-statistic) } & 0.045530 \\
\hline
\end{tabular}

Source: E-Views 7.0 
Appendix 9a: Niger's Regression Output of GDP $_{t-1}=$ 2.59-3.32INFLA t $_{t}+\operatorname{1.95INFLA}_{t-1}+U_{t}$

\begin{tabular}{|c|c|c|c|c|}
\hline \multicolumn{5}{|c|}{ Dependent Variable: $\mathrm{GDP}_{\mathrm{t}-1}$} \\
\hline \multicolumn{5}{|c|}{ Method: Least Squares } \\
\hline \multicolumn{5}{|c|}{ Date: $04 / 22 / 16$ Time: $15: 10$} \\
\hline \multicolumn{5}{|c|}{ Sample: 20012014} \\
\hline \multicolumn{5}{|c|}{ Included observations: 5} \\
\hline \multicolumn{5}{|c|}{ Excluded observations: 9} \\
\hline Variable & Coefficient & Std. Error & t-Statistic & Prob. \\
\hline INFLA & -3.323525 & 2.174941 & -1.528099 & 0.2661 \\
\hline INFLAT & 1.948565 & 1.891950 & 1.029924 & 0.4113 \\
\hline $\mathrm{C}$ & 2.590601 & 1.248047 & 2.075723 & 0.1736 \\
\hline R-squared & 0.791705 & \multicolumn{2}{|c|}{ Mean dependent var } & 2.800000 \\
\hline Adjusted R-squared & 0.583410 & \multicolumn{2}{|c|}{ S.D. dependent var } & 1.582688 \\
\hline S.E. of regression & 1.021526 & \multicolumn{2}{|c|}{ Akaike info criterion } & 3.164182 \\
\hline Sum squared resid & 2.087032 & \multicolumn{2}{|c|}{ Schwarz criterion } & 2.929845 \\
\hline Log likelihood & -4.910456 & \multicolumn{2}{|c|}{ F-statistic } & 3.800884 \\
\hline Durbin-Watson stat & 2.418517 & \multicolumn{2}{|c|}{ Prob(F-statistic) } & 0.208295 \\
\hline
\end{tabular}

Source: E-Views 7.0

\section{Appendix 9a: Niger's Regression Output of $\mathrm{UNEMPL}_{\mathrm{t}-1}=0.360+0.500 \mathrm{UR}_{\mathrm{t}-2}-0.003 I R$}

\begin{tabular}{|c|c|c|c|c|}
\hline \multicolumn{5}{|c|}{ Dependent Variable: $\mathrm{UR}_{\mathrm{t}-1}$} \\
\hline \multicolumn{5}{|c|}{ Method: Least Squares } \\
\hline \multicolumn{5}{|c|}{ Date: 02/29/16 Time: 15:05 } \\
\hline \multicolumn{5}{|c|}{ Sample: 20012014} \\
\hline \multicolumn{5}{|c|}{ Included observations: 14} \\
\hline Variable & Coefficient & Std. Error & t-Statistic & Prob. \\
\hline URT2 & 0.500295 & 0.162173 & 3.084954 & 0.0104 \\
\hline IR & -0.003159 & 0.011799 & -0.267729 & 0.7939 \\
\hline $\mathrm{C}$ & 0.360342 & 0.112120 & 3.213889 & 0.0082 \\
\hline R-squared & 0.475570 & \multicolumn{2}{|c|}{ Mean dependent var } & 0.656429 \\
\hline Adjusted R-squared & 0.380219 & \multicolumn{2}{|c|}{ S.D. dependent var } & 0.188990 \\
\hline S.E. of regression & 0.148784 & \multicolumn{2}{|c|}{ Akaike info criterion } & -0.785230 \\
\hline Sum squared resid & 0.243504 & \multicolumn{2}{|c|}{ Schwarz criterion } & -0.648289 \\
\hline Log likelihood & 8.496611 & \multicolumn{2}{|c|}{ F-statistic } & 4.987574 \\
\hline Durbin-Watson stat & 2.543691 & \multicolumn{2}{|c|}{ Prob(F-statistic) } & 0.028726 \\
\hline
\end{tabular}

Source: E-Views 7.0 
Appendix 10a: Senegal's Regression Output of GDP $_{t-1}=1.83+.55$ GDP $_{t-2}-0.0087$ INFLA $_{t-1}+U_{t}$

\begin{tabular}{|c|c|c|c|c|}
\hline \multicolumn{5}{|c|}{ Dependent Variable: $\mathrm{GDP}_{\mathrm{t}-1}$} \\
\hline \multicolumn{5}{|c|}{ Method: Least Squares } \\
\hline \multicolumn{5}{|c|}{ Date: $04 / 22 / 16$ Time: $15: 32$} \\
\hline \multicolumn{5}{|c|}{ Sample: 20012014} \\
\hline \multicolumn{5}{|c|}{ Included observations: 13} \\
\hline \multicolumn{5}{|c|}{ Excluded observations: 1} \\
\hline Variable & Coefficient & Std. Error & $\mathrm{t}$-Statistic & Prob. \\
\hline $\mathrm{GDP}_{\mathrm{t}-2}$ & 0.551767 & 0.177280 & 3.112403 & 0.0110 \\
\hline INFLA $_{t-2}$ & -0.008683 & 0.946328 & -0.009175 & 0.9929 \\
\hline $\mathrm{C}$ & 1.833870 & 0.582432 & 3.148645 & 0.0104 \\
\hline R-squared & 0.546946 & \multicolumn{2}{|c|}{ Mean dependent var } & 3.693846 \\
\hline Adjusted R-squared & 0.456336 & \multicolumn{2}{|c|}{ S.D. dependent var } & 1.122167 \\
\hline S.E. of regression & 0.827414 & \multicolumn{2}{|c|}{ Akaike info criterion } & 2.658151 \\
\hline Sum squared resid & 6.846141 & \multicolumn{2}{|c|}{ Schwarz criterion } & 2.788524 \\
\hline Log likelihood & -14.27798 & \multicolumn{2}{|c|}{ F-statistic } & 6.036223 \\
\hline Durbin-Watson stat & 2.785870 & \multicolumn{2}{|c|}{$\operatorname{Prob}(F-$-statistic) } & 0.019087 \\
\hline
\end{tabular}

Source: E-Views 7.0

Appendix 9a: Niger's Regression Output of $U N E M P L=0.974+0.193 U_{t-1}-0.198 U R_{t-2}-0.006 I R+U_{t}$

\begin{tabular}{|c|c|c|c|c|}
\hline \multicolumn{5}{|c|}{ Dependent Variable: UR } \\
\hline \multicolumn{5}{|c|}{ Method: Least Squares } \\
\hline \multicolumn{5}{|c|}{ Date: $02 / 29 / 16 \quad$ Time: $15: 13$} \\
\hline \multicolumn{5}{|c|}{ Sample: 20012014} \\
\hline \multicolumn{5}{|c|}{ Included observations: 12} \\
\hline \multicolumn{5}{|c|}{ Excluded observations: 2} \\
\hline Variable & Coefficient & Std. Error & t-Statistic & Prob. \\
\hline $\mathrm{UR}_{\mathrm{t}-1}$ & 0.192781 & 0.056775 & 3.395542 & 0.0094 \\
\hline $\mathrm{UR}_{\mathrm{t}-2}$ & -0.198135 & 0.042812 & -4.628019 & 0.0017 \\
\hline IR & -0.006430 & 0.045633 & -0.140903 & 0.8914 \\
\hline $\mathrm{C}$ & 0.973620 & 0.045750 & 21.28131 & 0.0000 \\
\hline R-squared & 0.734335 & \multicolumn{2}{|c|}{ Mean dependent var } & 0.962500 \\
\hline Adjusted R-squared & 0.634711 & \multicolumn{2}{|c|}{ S.D. dependent var } & 0.069167 \\
\hline S.E. of regression & 0.041804 & \multicolumn{2}{|c|}{ Akaike info criterion } & -3.250448 \\
\hline Sum squared resid & 0.013981 & \multicolumn{2}{|c|}{ Schwarz criterion } & -3.088812 \\
\hline Log likelihood & 23.50269 & \multicolumn{2}{|c|}{ F-statistic } & 7.371053 \\
\hline Durbin-Watson stat & 1.513572 & \multicolumn{2}{|c|}{ Prob(F-statistic) } & 0.010874 \\
\hline
\end{tabular}

Source: E-Views 7.0 


\section{REFERENCES}

[1] Abachi, T. (1998). Inflation - Unemployment Trade-off in Less Developed Countries (LDCs): The case study of Nigeria. Unpublished M.Sc. Thesis, Economics Department, Ahmadu Bello University $(A B U)$, Zaria

[2] Adamson, Y. (2000). Structural Disequilibrum and Inflation in Nigeria: A Theoretical and Empirical Analysis. Centre for Economic Research on Africa, New Jersey 07043: Montclair State University, Upper Montclair .

[3] Balami, G. (2006). Innovation and Growth in Developing Countries. Journal of Development Economics, July; Growth in Developing Countries, Journal of Economic Literature, September: Growth in the Global Economy. Cambridge, Mass: MIT Press, 33(3).

[4] Buhari, A. (1987). Straight to the point, . ICAN Economics, Unilorin Press, University of ilorin, Nigeria .

[5] CBN (2004) Annual Report and Statement of Account. Abuja: Central Bank of Nigeria.

[6] Friedman, M. (1976). Inflation and Unemployment. Chicago Journals - Journal of Political Economy, 451 - 472.

[7] Friedman, M. (1968). The Role of Monetary Policy. American Economic Review58, 1-17.

[8] Granger, C. (1969). Investigating casual relationships by econometric models and cross spectral methods. Econometrica, 17(2): 424-438.

[9] Hamilton, A. (2001). Exploding Inflation. Zea; Intelligence .

[10] Kamin, S., \& Klau, M. (1998). Some Multi-Country Evidence on the Effects of real Exchange Rates on Output. International Finance Discussion Papers, Washington,D.C: Federal Reserve Board, 611 .

[11] Jhingan, M.L (2008). Advanced Economics Theories: Vrinda (P) Ltd, New Delhi

[12] Hover. D (2016) New Classical Macroeconomics:The Concise Encyclopedia of Economics 2nd Edition () from www.econlib.org 23/04/2016
[13] Rational Expectation in the economy and Unemployment. (access online from www.Study.com) on 23/04/2016

[14] Omoke, P., \& Ugwuanyi, C. (2010). Money, Price and Output: A casuality Test for Nigeria. American Journal of Scientific Research ISSN 1456-223X, Issue 8, , pp. 78 - 87 Euro Journals Publishin, Inc.

[15] Orji, A., Orji-Anthony, I., \& Okafor, J. (2015). Inflation and Unemployment Nexus in Nigeria: Another test of the Phillip's Curve. . Asian Economic and Financial Review .

[16] Phillips, W. (1958). The Relationship between Unemployment and the Rate of Change of Money Wage Rates in the United Kingdom. Economica .

[17] Piana, V. (2001). Inflation Economics. Web Institute

[18] Popovic, G., \& Popovic, J. (2009). Inflation and Unemployment in the EU: Comparative Analysis of Phillips Regularity. UDK 336.748.12,331.56. Dyanamics in Nigeria: Is there Evidence of Jobless Growth? Paper presented at the 53rd Annual Conference of the Nigerian Economic Society (NES) on "Youth Employment and Poverty Reduction in Nigeria" Nicon Luxury Hotel, Abuja .

[19] Sack, D. (2016). Regional Economic Integration in West Africa. Center for Research on Political Economy.

[20] Stock, J., \& Watson, M. W. (1999). Forecasting Inflation. National Bureau of Economic Research (NBER) Working Paper 7023, March.

[21] WWW.Study.com: Rational Expectation in the economy and Unemployment. (access online from www.Study.com) on $23 / 04 / 2016$

[22] Umaru, A., \& Zubairu, A. (2012). An Empirical Analysis of the Relationship between Unemployment and inflation in Nigeria from 1977-2009. Economics and Finance Review Vol.1 .

[23] Wallich, H. (1979). Balancing Inflation and Unemployment. Controversies: Limits to Inflation?

[24] Williams, O., \& Adedeji. (2004). Inflation Dynamics in the Dominican Republic. IMF Working Paper, WP/04/29, Western Hemisphere Department: Washinton,D.C .

[25] World Bank. (1998). World Development Indicators. Washington, D.C. The World Bank. 